\title{
Antifreeze in the hot core of Orion
}

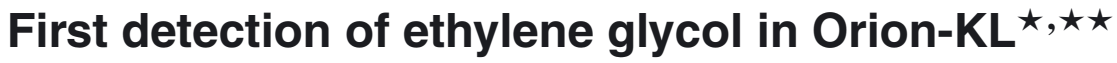

\author{
N. Brouillet ${ }^{1,2}$, D. Despois ${ }^{1,2}$, X.-H. Lu ${ }^{3,4}$, A. Baudry ${ }^{1,2}$, J. Cernicharo ${ }^{5}$, D. Bockelée-Morvan ${ }^{6}$, \\ J. Crovisier ${ }^{6}$, and N. Biver ${ }^{6}$
}

1 Univ. Bordeaux, LAB, UMR 5804, 33270 Floirac, France

e-mail: brouillet@obs.u-bordeaux1.fr

2 CNRS, LAB, UMR 5804, 33270 Floirac, France

3 Université de Toulouse, UPS-OMP, IRAP, 31028 Toulouse Cedex 4, France

4 CNRS, IRAP, 9 Av. Colonel Roche, BP 44346, 31028 Toulouse Cedex 4, France

5 Centro de Astrobiología (CSIC-INTA), Ctra de Torrejón a Ajalvir, km 4, 28850 Torrejón de Ardoz, Madrid, Spain

${ }^{6}$ LESIA, Observatoire de Paris, CNRS, UPMC, Université Paris-Diderot, 5 place Jules Janssen, 92195 Meudon, France

Received 11 July 2014 / Accepted 4 February 2015

\section{ABSTRACT}

\begin{abstract}
Context. Ices are present in comets and in the mantles of interstellar grains. Their chemical composition has been indirectly derived by observing molecules released in the gas phase, when comets approach the sun and when ice mantles are sublimated or destroyed, e.g. in the hot cores present in high-mass, star-forming regions. Comparison of these chemical compositions sheds light on the formation of comets and on the evolution of interstellar matter from the molecular cloud to a protoplanetary disk, and it shows, to first order, a good agreement between the cometary and interstellar abundances. However, a complex O-bearing organic molecule, ethylene gly$\mathrm{col}\left(\mathrm{CH}_{2} \mathrm{OH}\right)_{2}$, seems to depart from this correlation because it was not easily detected in the interstellar medium (Sgr B2) although it proved to be rather abundant with respect to other O-bearing species in comet C/1995 O1 (Hale-Bopp). Ethylene glycol thus appears, together with the closely related molecules glycolaldehyde $\mathrm{CH}_{2} \mathrm{OHCHO}$ and ethanol $\mathrm{CH}_{3} \mathrm{CH}_{2} \mathrm{OH}$, as a key species in the comparison of interstellar and cometary ices as well as in any discussion on the formation of cometary matter.

Aims. It is important to measure the molecular abundances in various hot cores to see if the observed differences between the interstellar medium and the comets are general. We focus here on the analysis of ethylene glycol in the nearest and best studied hot core-like region, Orion-KL.

Methods. We use ALMA interferometric data because high spatial resolution observations allow us to reduce the line confusion problem with respect to single-dish observations since different molecules are expected to exhibit different spatial distributions. Furthermore, a large spectral bandwidth is needed because many individual transitions are required to securely detect large organic molecules. Confusion and continuum subtraction are major issues and have been handled with care.

Results. We have detected the $a G g^{\prime}$ conformer of ethylene glycol in Orion-KL. The emission is compact and peaks towards the hot core close to the main continuum peak, about $2^{\prime \prime}$ to the south-west; this distribution is notably different from other O-bearing species. Assuming optically thin lines and local thermodynamic equilibrium, we derive a rotational temperature of $145 \pm 30 \mathrm{~K}$ and a column density of $4.6 \pm 0.8 \times 10^{15} \mathrm{~cm}^{-2}$. The limit on the column density of the $g G g^{\prime}$ conformer is five times lower.
\end{abstract}

Key words. astrochemistry - ISM: molecules - radio lines: ISM - ISM: individual objects: Orion-KL - comets: general

\section{Introduction}

The good global correlation between the chemical abundances in cometary ices and molecular hot cores (see Fig. 3 of Bockelée-Morvan et al. 2000) is a strong argument in favour of similar formation processes in these two rather different types of objects. Ion-molecule and surface reactions at a low $(<200 \mathrm{~K})$ temperature are thus invoked (Herbst \& van Dishoeck 2009) but one may not yet exclude the possibility of a very direct connection implying comets as being "frozen pieces of the interstellar

* Based on observations carried out with ALMA and the IRAM Plateau de Bure Interferometer. IRAM is supported by INSU/CNRS (France), MPG (Germany) and IGN (Spain).

$\star \star$ Appendix $\mathrm{A}$ is available in electronic form at http://www. aanda.org medium" as discussed as early as 1975 by Shimizu (see also Despois 1992, and references therein; and Mumma \& Charnley 2011, for a recent review).

However, at least two important problems arise: the $\mathrm{D} / \mathrm{H}$ ratio (e.g. Ceccarelli et al. 2014), and the crystalline/amorphous ratio in silicates do not seem to match well (e.g. Crovisier et al. 1997). Ethylene glycol may be a third example of a discrepancy. This opens the possibility for a wide range of models, ranging from pure interstellar matter to pure products of nebular chemistry, to explain the formation of comets. Consequently, different models could explain the protosolar nebula and solar system formation (see e.g. Irvine et al. 2000). This in turn has consequences on the way the collapse proceeds in young stellar objects, on physical and chemical evolution and timescales for the collapse and pre-collapse phase and later mixing in the disk due to turbulence (Bockelée-Morvan et al. 2002). 
Ethylene glycol, $\mathrm{HOCH}_{2}-\mathrm{CH}_{2} \mathrm{OH}$, is a dialcohol, a molecule chemically related to standard alcohol (ethanol, $\mathrm{CH}_{3}-\mathrm{CH}_{2} \mathrm{OH}$ ). Ethylene glycol is also known as an antifreeze coolant for car engines. Ethylene glycol was first found in the interstellar medium towards the Galactic centre along the line of sight to Sgr B2 despite line blending problems (Hollis et al. 2002; Requena-Torres et al. 2008). In comets, it has been identified for the first time in the Comet C/1995 O1 (Hale-Bopp) spectra by Crovisier et al. (2004a).

The detailed comparison of the abundances of ethylene glycol in the interstellar medium and in comets reveals a very different situation:

- In Hale-Bopp, ethylene glycol is rather abundant $(0.25 \%$ by number with respect to $\mathrm{H}_{2} \mathrm{O}$, or $1 \%$ by mass), just behind the main $\mathrm{CHO}$ species, methanol $\mathrm{CH}_{3} \mathrm{OH}(2.5 \%$ by number), and comparable to formaldehyde $(0.1-1.1 \%$ by number). Ethylene glycol has recently been detected in two other comets: C/2012 F6 (Lemmon) and C/2013 R1 (Lovejoy), with similar abundances relative to water $(0.24$ and $0.33 \%$, Biver et al. 2014). The simpler ethanol is not detected $(<0.1 \%-3 \sigma$ limit $)$, nor the companion molecule to ethylene glycol, glycolaldehyde $\left(\mathrm{HOCH}_{2}-\mathrm{CHO} ;<0.04 \%\right.$; Crovisier et al. 2004b).

- In the interstellar medium, ethylene glycol is not easy to identify, and until now it has been found towards only a few sources: Sgr B2 (Hollis et al. 2002; Requena-Torres et al. 2008), NGC 7129 FIRS 2 (Fuente et al. 2014), and NGC 1333-IRAS2A (Maury et al. 2014). Along the Sgr B2 line of sight, glycolaldehyde is seen with a similar abundance (within a factor of 2, Hollis et al. 2002). Ethanol is more abundant by an order of magnitude in Sgr B2 (Nummelin et al. 2000), and seen in many sources.

How can these differences be explained? Is the line of sight towards the galactic centre atypical, or are we seeing general differences between interstellar and cometary ices? Observations of hot cores, which appear to be the interstellar regions most similar to comets, are required to answer these questions.

Orion-KL is one of the main targets to search for new molecules in the interstellar medium, because of its molecular wealth and its proximity. Interferometric maps are absolutely needed because they notably reduce the confusion problem due to line blends in the single dish spectra. As shown by Wright et al. (1996) and confirmed by our data (Guélin et al. 2008; Favre et al. 2011; Peng et al. 2012, 2013; Brouillet et al. 2013), different sets of molecules peak towards different positions in Orion-KL, and possibly even do not overlap on high (1-2") spatial resolution maps. A second clear advantage of interferometer maps, is the information they provide: if the spatial distribution is identical for various lines, they most likely arise from the same species and not from a single interloper exhibiting the same frequencies as those of the molecular species under study. This is especially true for ethylene glycol, a molecule with a complex microwave spectrum.

In this paper, we report results of our search for the two most stable conformers of ethylene glycol towards Orion-KL. The spatial distribution of the ethylene glycol emission and the derived physical parameters are presented in Sect. 3. In Sect. 4 we discuss the chemistry in comparison to other interstellar sources and comets. We present the continuum emission map and its variation at nearby frequencies in Sect. 5. Finally our conclusions are summarized in Sect. 6.

\section{Observations}

We primarily use here the ALMA science verification (ALMA-SV) data ${ }^{1}$ taken on 20 January 2012 towards the IRc2 region in Orion. The observations were carried out with $16 \times 12 \mathrm{~m}$ antennas in the Band 6 frequency range from 213.715 to $246.627 \mathrm{GHz}$. 20 slightly overlapping spectral windows were observed, each with a $1.875 \mathrm{GHz}$ bandwidth and 3840 channels resulting in a $0.488 \mathrm{MHz}$ channel spacing corresponding to a velocity separation of $0.64 \mathrm{~km} \mathrm{~s}^{-1}$. The observations were centred on coordinates $\left(\alpha_{\mathrm{J} 2000}=05^{\mathrm{h}} 35^{\mathrm{m}} 14.35\right.$, $\delta_{\mathrm{J} 2000}=-05^{\circ} 22^{\prime} 35^{\prime \prime}$.00) with a primary beam of $\sim 27^{\prime \prime}$.

We used the CASA software ${ }^{2}$ for initial processing, and then we exported visibilities to the GILDAS package ${ }^{3}$ for further analysis. The line maps were cleaned using the CLARK algorithm (Clark 1980). The synthesized beam is $1.90^{\prime \prime} \times 1.40^{\prime \prime}$ with a PA of $170^{\circ}$ and the brightness temperature to flux density conversion factor is $9 \mathrm{~K}$ for $1 \mathrm{Jy}$ per beam. The continuum emission was subtracted in the maps by carefully selecting line-free channels (see Sect. 5).

We also analyzed several observational data sets obtained with the IRAM Plateau de Bure Interferometer (see Table 1 in Favre et al. 2011). These observations cover about $2 \mathrm{GHz}$ from $80.5 \mathrm{GHz}$ to $226 \mathrm{GHz}$ with a velocity separation ranging from $0.42 \mathrm{~km} \mathrm{~s}^{-1}$ to $2.33 \mathrm{~km} \mathrm{~s}^{-1}$ and a spatial resolution ranging from $1.79^{\prime \prime} \times 0.79^{\prime \prime}$ to $7.63^{\prime \prime} \times 5.35^{\prime \prime}$, depending on the frequency and the IRAM interferometer configuration. Four data sets correspond to frequencies included in the ALMA-SV data. In particular, the IRAM 223.4-223.9 GHz observations have spectral and spatial resolution and sensitivity similar to those in the ALMA data set for the same spectral range.

\section{Results}

\subsection{Conformers of ethylene glycol and selection of lines}

Ethylene glycol is an asymmetric top molecule with coupled rotation around its $\mathrm{C}-\mathrm{C}$ bond and its two $\mathrm{C}-\mathrm{O}$ bonds leading to different spatial arrangements or conformers (see Christen et al. 1995, 2001). There are six conformers relative to the $\mathrm{C}-\mathrm{C}$ bond with gauche $(\mathrm{G})$ arrangement of the two ethylene groups and four less stable conformers with anti-arrangement of the $\mathrm{CH}_{2}$ groups. In the $\mathrm{G}$ arrangement, the two $\mathrm{OH}$ groups adopt the gauche orientation with respect to each other. The notations a and g are used for the anti (a) and gauche (g) orientations of the $\mathrm{OH}$ groups with respect to the two $\mathrm{C}-\mathrm{O}$ bonds. When the $\mathrm{OH}$ group provides the hydrogen intramolecular bonding, the gauche orientation is noted $g^{\prime}$. There are only two conformers with intramolecular bonding, $a G g^{\prime}$ and $g G g^{\prime}$, and Müller \& Christen (2004) showed that the latter lies about $200 \mathrm{~cm}^{-1}$ or about $290 \mathrm{~K}$ higher in energy than $a G g^{\prime}$. For these two conformers, tunneling is observed between two equivalent equilibrium configurations and splits each rotational level into two distinct states designated $v=0$ and $v=1$ (e.g. Christen et al. 2001). The $v=1$ state is around 7 and $1.4 \mathrm{GHz}$ higher than the $v=0$ state for the $a G g^{\prime}$ and $g G g^{\prime}$ conformers, respectively (Christen \& Müller 2003).

The rotation-tunnelling microwave spectra of the $a G g^{\prime}$ and $g G g^{\prime}$ conformers were accurately determined in the 54-370 GHz (Christen \& Müller 2003) and 77-579 GHz (Müller $\&$ Christen 2004) ranges, respectively. The results from these

\footnotetext{
1 http://almascience.eso.org/almadata/sciver/ OrionKLBand6/

2 http://casa.nrao.edu

3 http://www . iram. fr/IRAMFR/GILDAS
} 


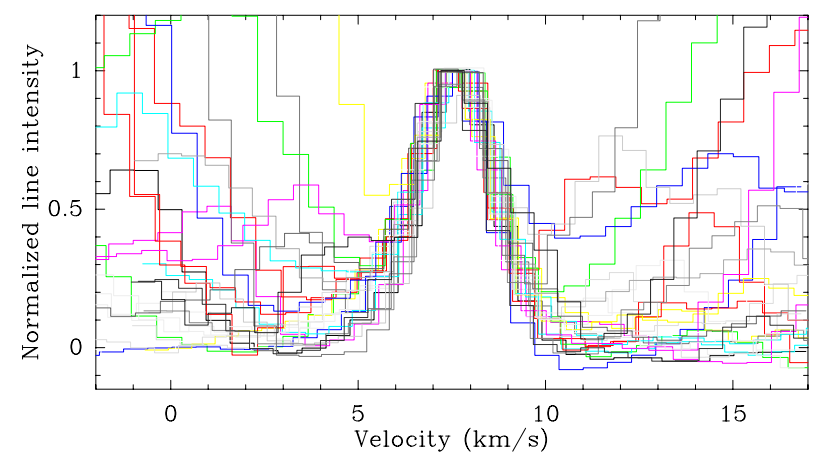

Fig. 1. Superposition of the unblended and partially blended ethylene glycol lines listed in Table 1. The lines corresponding to close pairs of transitions separated by $0.5-1 \mathrm{MHz}$ are not included in this plot as their profile is broadened. The spectra are towards the ethylene glycol peak $\left(\alpha_{\mathrm{J} 2000}=05^{\mathrm{h}} 35^{\mathrm{m}} 14.47, \delta_{\mathrm{J} 2000}=-05^{\circ} 22^{\prime} 33^{\prime \prime}{ }^{\prime} 17\right)$ and are normalized to 1 .

works and earlier works are now available from the Cologne database for molecular spectroscopy $\left(\mathrm{CDMS}^{4}\right.$, Müller et al. 2001, 2005) and can be used for reliable identification of the ethylene glycol transitions. Earlier ethylene glycol line predictions were used to assign to the $a G g^{\prime}$ conformer five features observed by Hollis et al. (2002) in Sgr B2; two other features were also assigned to the same conformer in the same source by Requena-Torres et al. (2008). The CDMS was used to identify more than $10 a G g^{\prime}$ conformer lines in the Hale-Bopp comet (Crovisier et al. 2004a). We note that several ethylene glycol transitions observed in the direction of Sgr B2 are blended, while transitions are well "isolated" towards Hale-Bopp.

We used the most recent CDMS data to search for transitions for the $a G g^{\prime}$ and $g G g^{\prime}$ conformers in the ALMA-SV data cube. From line intensity predictions at $120 \mathrm{~K}$, a typical temperature for the region (e.g. Favre et al. 2011), we have examined the strongest lines among the ethylene glycol transitions expected in the different spectral bands. We produced synthetic spectra for both conformers (see Sects. 3.4 and 3.5). We also derived synthetic spectra for higher temperatures of the $g G g^{\prime}$ conformer to investigate the presence of the $g G g^{\prime}$ conformer in hotter gas because $g G g^{\prime}$ is higher in energy than $a G g^{\prime}$ (see above).

\subsection{Spatial distribution and line profile of the $a G g^{\prime}$ conformer of ethylene glycol}

In the ALMA dataset, most ethylene glycol lines suffer mild to heavy blending. Table 1 lists 57 transitions that are mildly blended and a noncontaminated transition lying at $228752 \mathrm{MHz}$. All these transitions present a peak in their spatial distributions at $\left(\alpha_{\mathrm{J} 2000}=05^{\mathrm{h}} 35^{\mathrm{m}} 14.47, \delta_{\mathrm{J} 2000}=-05^{\circ} 22^{\prime} 33^{\prime \prime} \cdot 17\right)$. In Fig. 1 we plotted a superposition of the spectra of the partially blended ethylene glycol lines towards this position, excluding line doublets with a difference in frequency of $\sim 0.5-1 \mathrm{MHz}$. We normalized the spectra to a peak intensity of 1 . All line profiles are similar in the 5 to $10 \mathrm{~km} \mathrm{~s}^{-1}$ velocity range. They can be fitted with a Gaussian centred at a velocity of $7.6 \mathrm{~km} \mathrm{~s}^{-1}$ and a line width of $2.3 \mathrm{~km} \mathrm{~s}^{-1}$. We have averaged the transitions that exhibit no blends around $5 \mathrm{~km} \mathrm{~s}^{-1}$ on one hand, and the transitions that exhibit no blends around $10 \mathrm{~km} \mathrm{~s}^{-1}$ on the other hand. Figure 2 shows the superposition of these two spectra together with the $228752 \mathrm{MHz}$ transition spectrum. The resulting line profile of the unblended parts fits perfectly the profile of the

\footnotetext{
4 http://www.astro.uni-koeln.de/cdms
}

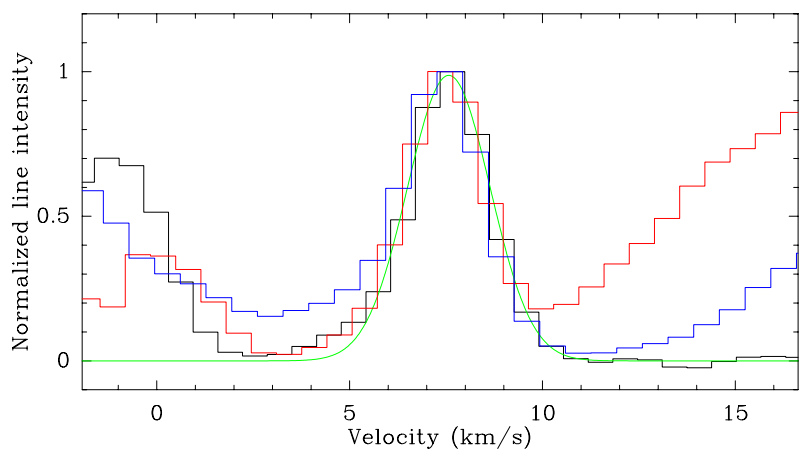

Fig. 2. Superposition of the $228752 \mathrm{MHz}$ transition spectrum at the ethylene glycol peak (in black) with the average spectrum of 11 transitions that have no blends near $5 \mathrm{~km} \mathrm{~s}^{-1}$ (in red) and the average spectrum of 14 transitions that have no blends near $10 \mathrm{~km} \mathrm{~s}^{-1}$ (in blue). All spectra are normalized to 1 . A Gaussian fit to the $228752 \mathrm{MHz}$ line is shown in green. A faint wing is visible at lower velocities.

$228752 \mathrm{MHz}$ transition. The Gaussian fit shows a faint excess around $5 \mathrm{~km} \mathrm{~s}^{-1}$. This excess is significant as it is above the noise of the $228752 \mathrm{MHz}$ individual spectrum. Furthermore it appears on both profiles, which use different transitions, shown in Fig. 2.

The spatial distribution of ethylene glycol in Orion-KL is shown in Fig. 3, which presents the intensity maps integrated over the noncontaminated channels of the five best lines (transitions at $219385 \mathrm{MHz}, 222348 \mathrm{MHz}, 228752 \mathrm{MHz}$, $231524 \mathrm{MHz}$, and $235834 \mathrm{MHz}$ ). The $228752 \mathrm{MHz}$ transition appears to be the only one free of contamination, i.e. without any blending line above the noise level; the channel maps are shown in Fig. 4.

The $a G g^{\prime}$ conformer distribution is compact and appears to be slightly bigger than the beam. We find an upper limit for the deconvolved size of $2.4^{\prime \prime} \times 1.1^{\prime \prime}$. The emission peak is located $\sim 2^{\prime \prime}$ to the south-west of the peak of continuum emission (see map in lower right panel of Fig. 3 and Fig. 8 map); it lies between the methyl formate MF2 and MF6 peaks of Favre et al. (2011). The line profile is similar to the methyl formate and ethanol line profiles at the same position, centred at a velocity of $\sim 7.6 \mathrm{~km} \mathrm{~s}^{-1}$ and with a faint wing around $5 \mathrm{~km} \mathrm{~s}^{-1}$. A detailed comparison of these O-bearing molecules will be performed in a forthcoming paper.

\subsection{Spectral confusion}

The spectral line density in Orion is high (e.g. 8.6 lines per $100 \mathrm{MHz}$ in the IRAM 30 m survey of Tercero et al. 2010), comparable to that in Sgr B2 (6-10 lines per $100 \mathrm{MHz}$, Friedel et al. 2004; Halfen et al. 2006) for high-sensitivity observations.

Snyder et al. (2005) have given the essential criteria for establishing the identification of a new interstellar molecule. Even if ethylene glycol has already been detected in Sgr B2 and two other interstellar sources, its identification is not obvious because of the weakness of the lines and spectral confusion. The different criteria are:

- the rest frequencies must be known with a high degree of accuracy from laboratory measurements;

- the central velocity and line width must agree for all detected transitions;

- blended lines must be separated by at least half the line width (FWHM) of the weaker line, to be considered as resolved; 
Table 1. Ethylene glycol transitions ( $a G g^{\prime}$ conformer) not blended or only partially blended in the ALMA dataset.

\begin{tabular}{|c|c|c|c|c|c|}
\hline $\begin{array}{l}\text { Frequency }{ }^{a} \\
(\mathrm{MHz})\end{array}$ & Quantum numbers ${ }^{b}$ & $\begin{array}{l}S \mu^{2 c} \\
\left(\mathrm{D}^{2}\right)\end{array}$ & $\log \left(A_{\mathrm{ul}}\right)^{d}$ & $\begin{array}{l}E_{\mathrm{u}}{ }^{d} \\
(\mathrm{~K})\end{array}$ & $g_{\mathrm{u}}{ }^{d}$ \\
\hline 214526.41410 & $19(11,8) v=1-19(10,9) v=1$ & 50.7 & -4.67073 & 153.1 & 273 \\
\hline 214526.41470 & $19(11,9) v=1-19(10,10) v=1$ & 65.2 & -4.67067 & 153.1 & 351 \\
\hline 214712.96650 & $21(5,16) v=0-20(5,15) v=1$ & 732.5 & -3.66141 & 127.1 & 387 \\
\hline 214808.01940 & $23(2,22) v=0-22(2,21) v=1$ & 685.7 & -3.61897 & 132.4 & 329 \\
\hline 214829.92520 & $23(1,22) v=0-22(1,21) v=1$ & 875.5 & -3.62187 & 132.4 & 423 \\
\hline $216685.81470^{*}$ & $21(3,19) v=1-20(3,18) v=0$ & 663.4 & -3.69253 & 117.3 & 387 \\
\hline 216826.11240 & $20(5,15) v=1-19(5,14) v=0$ & 557.3 & -3.74666 & 116.8 & 369 \\
\hline 217139.72350 & $21(4,17) v=0-20(4,16) v=1$ & 786.8 & -3.61568 & 123.9 & 387 \\
\hline 217449.99470 & $24(1,24) v=1-23(1,23) v=1$ & 928.6 & -3.59857 & 136.4 & 441 \\
\hline $217450.27020^{*}$ & $24(0,24) v=0-23(0,23) v=1$ & 722.2 & -3.59863 & 136.4 & 343 \\
\hline $217587.54780^{*}$ & $21(2,19) v=1-20(2,18) v=0$ & 666.2 & -3.57614 & 117.2 & 301 \\
\hline 219089.72000 & $22(10,13) v=0-21(10,12)) v=1$ & 670.7 & -3.69311 & 173.5 & 405 \\
\hline 219089.72790 & $22(10,12) v=0-21(10,11) v=1$ & 521.6 & -3.69317 & 173.5 & 315 \\
\hline $219384.91030^{*}$ & $26(11,16) v=1-26(10,16) v=0$ & 15.8 & -5.28218 & 232.4 & 371 \\
\hline $219385.17780^{*}$ & $22(9,14) v=0-21(9,13) v=1$ & 703.8 & -3.67045 & 164.3 & 405 \\
\hline $219385.32400^{*}$ & $26(11,15) v=1-26(10,17) v=0$ & 20.3 & -5.28213 & 232.4 & 477 \\
\hline $219385.42560^{*}$ & $22(9,13) v=0-21(9,12) v=1$ & 547.3 & -3.67051 & 164.3 & 315 \\
\hline 221007.82310 & $21(4,18) v=1-20(4,17) v=0$ & 818.3 & -3.57562 & 122.1 & 387 \\
\hline $221038.79970^{*}$ & $22(6,17) v=0-21(6,16) v=1$ & 771.6 & -3.62074 & 142.6 & 405 \\
\hline 221100.31520 & $22(5,18) v=0-21(5,17) v=1$ & 762.5 & -3.62553 & 137.4 & 405 \\
\hline $222054.23240^{*}$ & $21(10,12) v=1-20(10,11) v=0$ & 624.5 & -3.68686 & 163.0 & 387 \\
\hline $222054.23580^{*}$ & $21(10,11) v=1-20(10,10) v=0$ & 485.8 & -3.68681 & 163.0 & 301 \\
\hline $222348.60040^{*}$ & $23(1,23) v=1-22(1,22) v=0$ & 889.5 & -3.57018 & 126.0 & 423 \\
\hline $222349.15090^{*}$ & $23(0,23) v=1-22(0,22) v=0$ & 692.0 & -3.57003 & 126.0 & 329 \\
\hline $223741.66380^{*}$ & $21(6,16) v=1-20(6,15) v=0$ & 738.2 & -3.60437 & 132.0 & 387 \\
\hline $226643.30070^{*}$ & $25(1,25) v=0-24(1,24) v=1$ & 752.5 & -3.54420 & 147.7 & 357 \\
\hline $226643.45630^{*}$ & $25(0,25) v=0-24(0,24) v=1$ & 967.6 & -3.54415 & 147.7 & 459 \\
\hline 228602.83320 & $23(16,7) v=0-22(16,6) v=1$ & 456.4 & -3.82381 & 261.3 & 423 \\
\hline 228602.83320 & $23(16,8) v=0-22(16,7) v=1$ & 354.9 & -3.82387 & 261.3 & 329 \\
\hline $228752.77650^{*}, e$ & $23(2,22) v=1-22(2,21) v=0$ & 875.2 & -3.54020 & 132.7 & 423 \\
\hline 229233.71610 & $23(11,13) v=0-22(11,12) v=1$ & 530.2 & -3.64599 & 195.1 & 329 \\
\hline 229233.71660 & $23(11,12) v=0-22(11,11) v=1$ & 681.8 & -3.64594 & 195.1 & 423 \\
\hline $231127.40080^{*}$ & $23(7,16) v=0-22(7,15) v=1$ & 801.1 & -3.56515 & 160.2 & 423 \\
\hline $231524.03310^{*}$ & $23(6,18) v=0-22(6,17) v=1$ & 618.1 & -3.56638 & 154.1 & 329 \\
\hline 231564.00490 & $24(1,24) v=1-23(1,23) v=0$ & 722.1 & -3.51672 & 136.8 & 343 \\
\hline 231564.31950 & $24(0,24) v=1-23(0,23) v=0$ & 928.6 & -3.51666 & 136.8 & 441 \\
\hline 232095.73820 & $22(12,11) v=1-21(12,10) v=0$ & 462.3 & -3.67043 & 195.4 & 315 \\
\hline 232095.73830 & $22(12,10) v=1-21(12,9) v=0$ & 594.5 & -3.67038 & 195.4 & 405 \\
\hline 232349.45090 & $22(20,2) v=1-21(20,1) v=0$ & 146.9 & -4.27619 & 320.9 & 405 \\
\hline 232349.45090 & $22(20,3) v=1-21(20,2) v=0$ & 114.2 & -4.27614 & 320.9 & 315 \\
\hline 232350.05940 & $22(10,13) v=1-21(10,12) v=0$ & 522.1 & -3.61615 & 173.8 & 315 \\
\hline 232350.06810 & $22(10,12) v=1-21(10,11) v=0$ & 671.4 & -3.61610 & 173.8 & 405 \\
\hline 232881.53340 & $23(6,17) v=0-22(6,16) v=1$ & 750.3 & -3.58376 & 154.2 & 423 \\
\hline $235304.05030^{*}$ & $22(6,16) v=1-21(6,15) v=0$ & 773.7 & -3.53805 & 143.1 & 405 \\
\hline 235441.43720 & $10(7,4) v=0-9(6,3) v=0$ & 56.6 & -4.34165 & 51.1 & 189 \\
\hline 235441.48820 & $10(7,3) v=0-9(6,4) v=0$ & 44.1 & -4.34161 & 51.1 & 147 \\
\hline 235442.34320 & $10(7,4) v=1-9(6,3) v=1$ & 42.1 & -4.36183 & 51.4 & 147 \\
\hline 235442.39560 & $10(7,3) v=1-9(6,4) v=1$ & 54.1 & -4.36177 & 51.4 & 189 \\
\hline 235471.89630 & $8(8,0) v=0-7(7,1) v=0$ & 49.0 & -4.20372 & 49.1 & 119 \\
\hline 235471.89630 & $8(8,1) v=0-7(7,0) v=0$ & 63.0 & -4.20376 & 49.1 & 153 \\
\hline $235834.23970^{*}$ & $26(1,26) v=0-25(1,25) v=1$ & 1006.5 & -3.49194 & 159.3 & 477 \\
\hline $235834.32720^{*}$ & $26(0,26) v=0-25(0,25) v=1$ & 782.7 & -3.49199 & 159.3 & 371 \\
\hline $239605.33240^{*}$ & $24(11,14) v=0-23(11,13) v=1$ & 728.5 & -3.57758 & 206.9 & 441 \\
\hline $239605.33370^{*}$ & $24(11,13) v=0-23(11,12) v=1$ & 566.7 & -3.57753 & 206.9 & 343 \\
\hline 241545.26260 & $24(7,18) v=0-23(7,17) v=1$ & 841.5 & -3.50446 & 172.1 & 441 \\
\hline $242947.99050^{*}$ & $23(9,15) v=1-22(9,14) v=0$ & 749.2 & -3.52928 & 175.9 & 423 \\
\hline $242948.59120^{*}$ & $23(9,14) v=1-22(9,13) v=0$ & 582.8 & -3.52923 & 175.9 & 329 \\
\hline 243259.73980 & $24(6,18) v=0-23(6,17) v=0$ & 324.4 & -3.80007 & 166.2 & 343 \\
\hline 244685.14770 & $24(2,22) v=1-23(2,21) v=1$ & 939.2 & -3.43992 & 150.1 & 441 \\
\hline
\end{tabular}

Notes. ${ }^{(a)}$ Transitions used for the rotational diagram (see Fig. 5) are indicated by a star. ${ }^{(b)}$ The quantum numbers are $J\left(K_{\mathrm{a}}, K_{\mathrm{c}}\right)$ as defined in the CDMS database where $v$ is a state number (see Sect. 3.1). ${ }^{(c)} S$ is the line strength and $\mu$ the dipole moment. ${ }^{(d)}$ See Sect. 3.4 for the definition of the parameters. ${ }^{(e)}$ Not blended transition. 

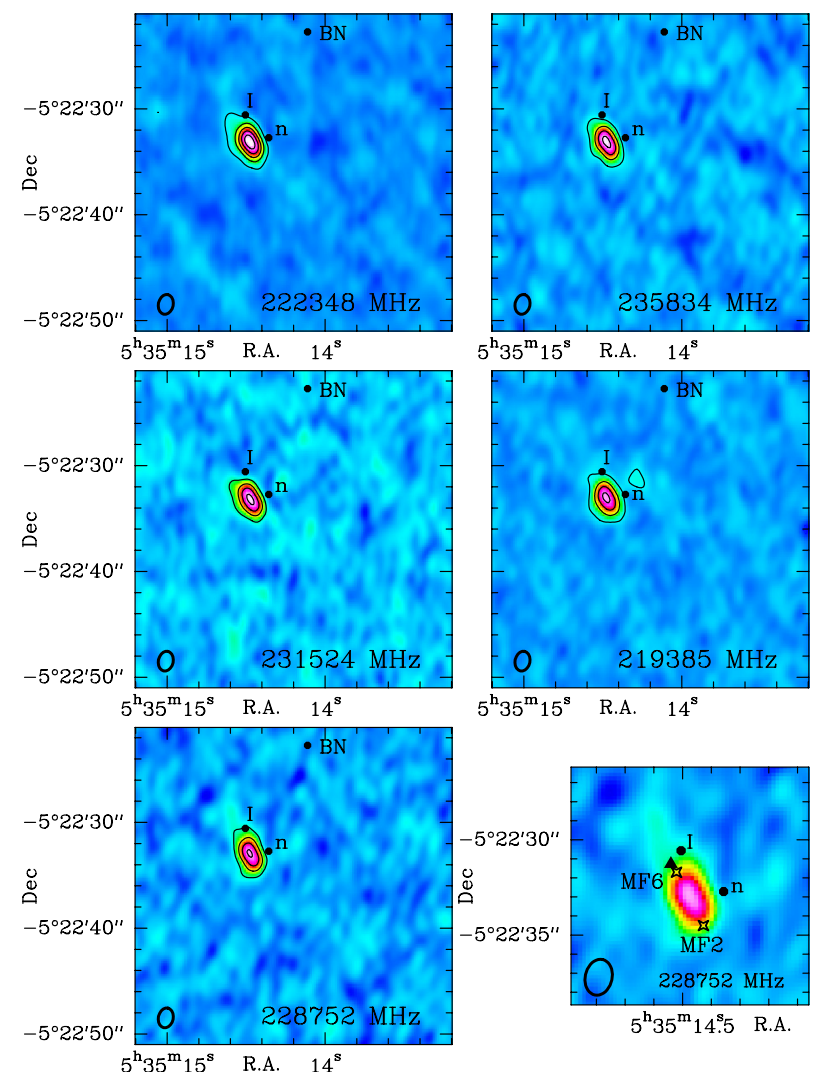

Fig. 3. ALMA ethylene glycol integrated intensity maps of the five less blended lines. The frequency of the transition is indicated at the bottom of the map and the first contour level corresponds to $4 \sigma$. The beam shown in the bottom left corner is $1.9^{\prime \prime} \times 1.39^{\prime \prime}$. The BN object position is $\left(\alpha_{\mathrm{J} 2000}=05^{\mathrm{h}} 35^{\mathrm{m}} 14^{\mathrm{s}} 11, \delta_{\mathrm{J} 2000}=-05^{\circ} 22^{\prime} 22^{\prime \prime} .72\right)$, the radio source I position is $\left(\alpha_{\mathrm{J} 2000}=05^{\mathrm{h}} 35^{\mathrm{m}} 14.51, \delta_{\mathrm{J} 2000}=-05^{\circ} 22^{\prime} 30^{\prime} \cdot 57\right)$, and the IR source n position is $\left(\alpha_{\mathrm{J} 2000}=05^{\mathrm{h}} 35^{\mathrm{m}} 14 \mathrm{~s} 36, \delta_{\mathrm{J} 2000}=-05^{\circ} 22^{\prime} 32^{\prime \prime}\right.$. 72$)$ (Goddi et al. 2011b). The map to the lower right is a blow up of the $228752 \mathrm{MHz}$ map. The triangle marks the position of the continuum emission peak (see map in Fig. 8) and the stars mark the MF2 and MF6 methyl formate positions of Favre et al. (2011). The ethylene glycol emission peaks at $\left(\alpha_{\mathrm{J} 2000}=05^{\mathrm{h}} 35^{\mathrm{m}} 14.47, \delta_{\mathrm{J} 2000}=-05^{\circ} 22^{\prime} 33^{\prime \prime} \cdot 17\right)$. The lines are integrated over the velocity range $5-10 \mathrm{~km} \mathrm{~s}^{-1}$ for the $222348 \mathrm{MHz}$ transition, $5.5-10 \mathrm{~km} \mathrm{~s}^{-1}$ for $235834 \mathrm{MHz}, 7-10 \mathrm{~km} \mathrm{~s}^{-1}$ for $231524 \mathrm{MHz}, 6-9.5 \mathrm{~km} \mathrm{~s}^{-1}$ for $219385 \mathrm{MHz}$ and $4-11 \mathrm{~km} \mathrm{~s}^{-1}$ for $228752 \mathrm{MHz}$. The level step and first contour are 6 and $2.8 \mathrm{~K} \mathrm{~km} \mathrm{~s}^{-1}$ for the $222348 \mathrm{MHz}$ transition, 7.6 and $3.6 \mathrm{~K} \mathrm{~km} \mathrm{~s}^{-1}$ for $235834 \mathrm{MHz}, 2.5$ and $1.7 \mathrm{~K} \mathrm{~km} \mathrm{~s}^{-1}$ for $231524 \mathrm{MHz}, 5$ and $1.9 \mathrm{~K} \mathrm{~km} \mathrm{~s}^{-1}$ for $219385 \mathrm{MHz}$ and 4.4 and $2.9 \mathrm{~K} \mathrm{~km} \mathrm{~s}^{-1}$ for $228752 \mathrm{MHz}$.

- beam dilution must be taken into account when comparing observations made at different frequencies;

- the relative intensities of the different transitions must be consistent (when, as we suppose it is the case here, an excitation close to local thermodynamic equilibrium is likely);

- all transitions with intensity predictions leading to detectable signal levels must be present.

For interferometric observations, another criterion can be added: the coherence of the spatial distribution. Indeed the spatial distribution of the different transitions must be similar for similar excitation conditions (e.g. for a similar upper level energy). The probability of chance alignment in frequency in one spectrum may be high, but a chance alignment for all spectra in an extended map is unlikely. As most of the molecules display different distributions on a high-resolution scale, confusion will not produce similar maps for every transition. Requiring

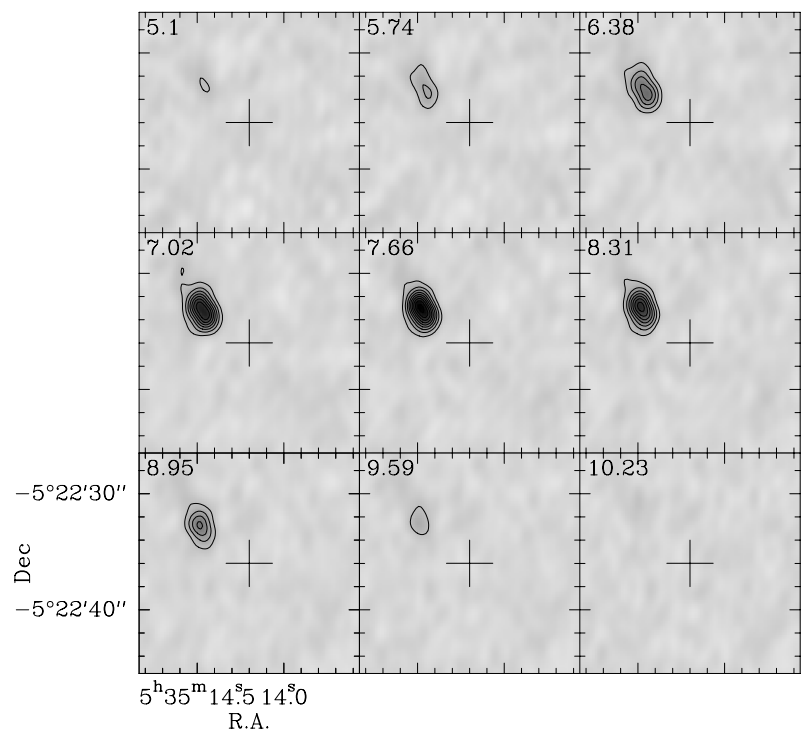

Fig. 4. ALMA channel maps of the ethylene glycol $228752 \mathrm{MHz}$ transition which has no apparent contamination. The level step and first contour are $0.44 \mathrm{~K}$. The LSR velocity in $\mathrm{km} \mathrm{s}^{-1}$ is indicated in the upper left corner of each map.

a single interloper for different transitions makes a misidentification much less probable.

Detailed study of the ethylene glycol spectroscopy is relatively recent and we can be confident that the rest frequencies are accurate (see Sect. 3.1). In our study of Orion-KL, there is a frequency agreement among all the partially blended transitions: the line centre velocities are in good agreement within the uncertainties $\left(7.6 \pm 0.2 \mathrm{~km} \mathrm{~s}^{-1}\right)$ as well as the line widths and the general profiles (see Fig. 1). In addition, the noncontaminated parts of these lines display the same spatial distribution (see Fig. 3). The lines that we consider partially blended with others are separated by at least their half-maximum intensity. The relative intensities of the transitions are consistent: no expected strong lines are missing and a rotational temperature can be estimated based on these transitions (see Sect. 3.4).

Halfen et al. (2006) add that the evidence for emission from a given molecule must be over a substantial frequency range and that an extensive, self-consistent data set is necessary to identify large organic species in the interstellar gas. Indeed, our initial search for ethylene glycol in Orion-KL based on limited spectral ranges with the IRAM Plateau de Bure Interferometer (PdBI), was not convincing enough. The ALMA-SV data are similar to our $223 \mathrm{GHz}$ PdBI data for their sensitivity and their spectral and spatial resolution but they cover $32.9 \mathrm{GHz}$ instead of 0.5 GHz. Using all the PdBI data sets (Favre et al. 2011), which cover $\sim 2 \mathrm{GHz}$, we have identified seven blended lines coinciding with $a G g^{\prime}$ conformer transitions ${ }^{5}$. However, we have discarded these lines from the present analysis because of line blending problems and because of different spatial and spectral resolution used in our PdBI data.

\subsection{Physical parameters of the $a G g^{\prime}$ conformer of ethylene glycol}

Assuming optically thin lines and local thermodynamic equilibrium, we have estimated the rotational temperature and the

\footnotetext{
5 Frequencies of the PdBI ethylene glycol blended lines: $105.7010 \mathrm{GHz}, 105.8347 \mathrm{GHz}, 203.4309 \mathrm{GHz}, 203.4533 \mathrm{GHz}$, 223.4589 GHz, 223.5205 GHz, 223.7417 GHz.
} 


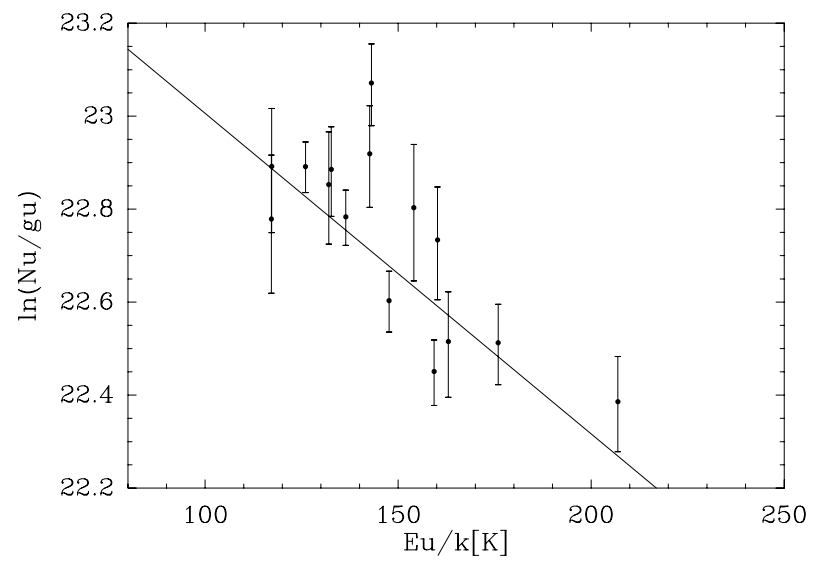

Fig. 5. Rotational diagram at the emission peak based on the lesscontaminated ethylene glycol lines indicated by a star in Table 1.

column density of ethylene glycol using the equation (e.g. Goldsmith \& Langer 1999):

$\ln \left(\frac{8 \pi k_{B} v^{2} W}{h c^{3} g_{\mathrm{u}} A_{\mathrm{ul}}}\right)=\ln \left(\frac{N}{Q(T)}\right)-\frac{E_{\mathrm{u}}}{k_{B} T}$,

where $W$ is the integrated line intensity $\left(\mathrm{K} \mathrm{km} \mathrm{s}^{-1}\right), v$ the line frequency $(\mathrm{Hz}), A_{\mathrm{ul}}$ the Einstein coefficient for spontaneous emission $\left(\mathrm{s}^{-1}\right), g_{\mathrm{u}}$ the degeneracy of the upper state, $N$ the total column density, $Q(T)$ the partition function, $E_{\mathrm{u}}$ the upper state energy, and $T$ the excitation temperature.

The rotational diagram is obtained at the emission peak from the less-contaminated ethylene glycol lines to derive reliable physical parameters (see Fig. 5). Taking the partition function $Q(T)=234500$ from the nonlinear plot of the CDMS values of $Q$ at $145 \mathrm{~K}$, we obtain a rotational temperature of $145 \pm 30 \mathrm{~K}$ and derive a total column density averaged over the synthesized beam $N_{a G g^{\prime}}$ of $4.6 \pm 0.8 \times 10^{15} \mathrm{~cm}^{-2}$. From the continuum map (see Sect. 5), we derived the beam averaged projected density $N_{\mathrm{H}_{2}}$ as in Favre et al. (2011), and we find an ethylene glycol abundance of $\sim 10^{-9}$.

We calculated the synthetic spectrum for a velocity $v_{\mathrm{LSR}}$ of $7.6 \mathrm{~km} \mathrm{~s}^{-1}$, a linewidth $\Delta v_{1 / 2}$ of $2.3 \mathrm{~km} \mathrm{~s}^{-1}$, a column density $N_{a G g^{\prime}}$ of $4.6 \times 10^{15} \mathrm{~cm}^{-2}$ and a temperature $T$ of $145 \mathrm{~K}$. Figure 6 shows the synthetic spectrum overlaid on the observed ALMA spectrum towards the ethylene glycol peak in the range 228.74-228.79 GHz. Figure 7 shows the observed and synthetic spectra of the ethylene glycol transitions listed in Table 1. The synthetic spectrum overlaid to the whole ALMA band spectrum is shown in Fig. A.1. Figure 6 shows that the transition at $228752 \mathrm{MHz}$ is not blended, while the two other lines around 228778 and $228782 \mathrm{MHz}$ show line contamination and are not included in Table 1.

\subsection{Search for the gGg' conformer of ethylene glycol}

Because the $g G g^{\prime}$ conformer of ethylene glycol is not yet detected in the interstellar medium, we also searched for transitions from this stable conformer although it is $200 \mathrm{~cm}^{-1}(290 \mathrm{~K})$ higher in energy than the $a G g^{\prime}$ conformer (Müller \& Christen 2004).

All expected strong lines appear to be blended in the ALMA data cube used in this study. Making the simplifying, but admittedly questionable, hypotheses that the $g G g^{\prime}$ conformer presents the same spatial distribution and the same temperature as the $a G g^{\prime}$ conformer, the comparison of the $g G g^{\prime}$ synthetic spectrum

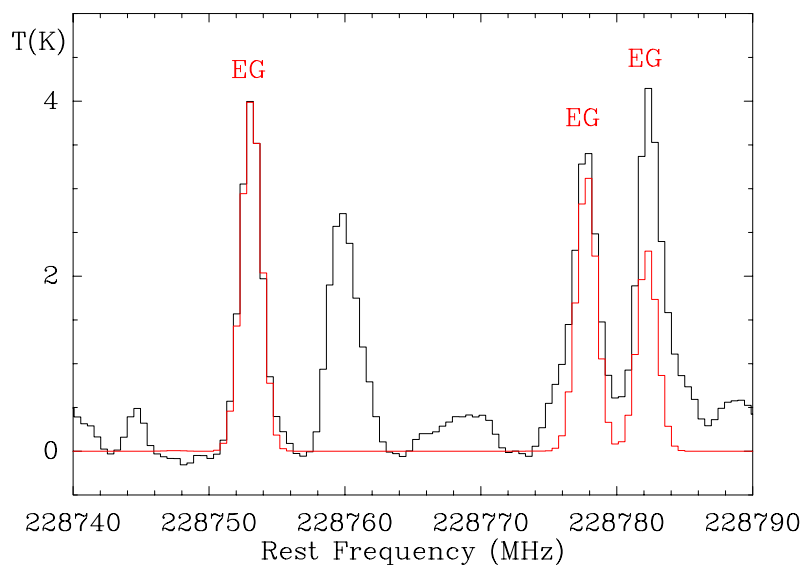

Fig. 6. Part of the ALMA spectrum (in black) towards the ethylene glycol peak. The synthetic spectrum of ethylene glycol (EG) is overlaid in red. The parameters used for the synthetic spectrum are: $v_{\mathrm{LSR}}=$ $7.6 \mathrm{~km} \mathrm{~s}^{-1}, \Delta v_{1 / 2}=2.3 \mathrm{~km} \mathrm{~s}^{-1}, N_{a G g^{\prime}}=4.6 \times 10^{15} \mathrm{~cm}^{-2}, T=145 \mathrm{~K}$. Only the line on the left is devoid of confusion.
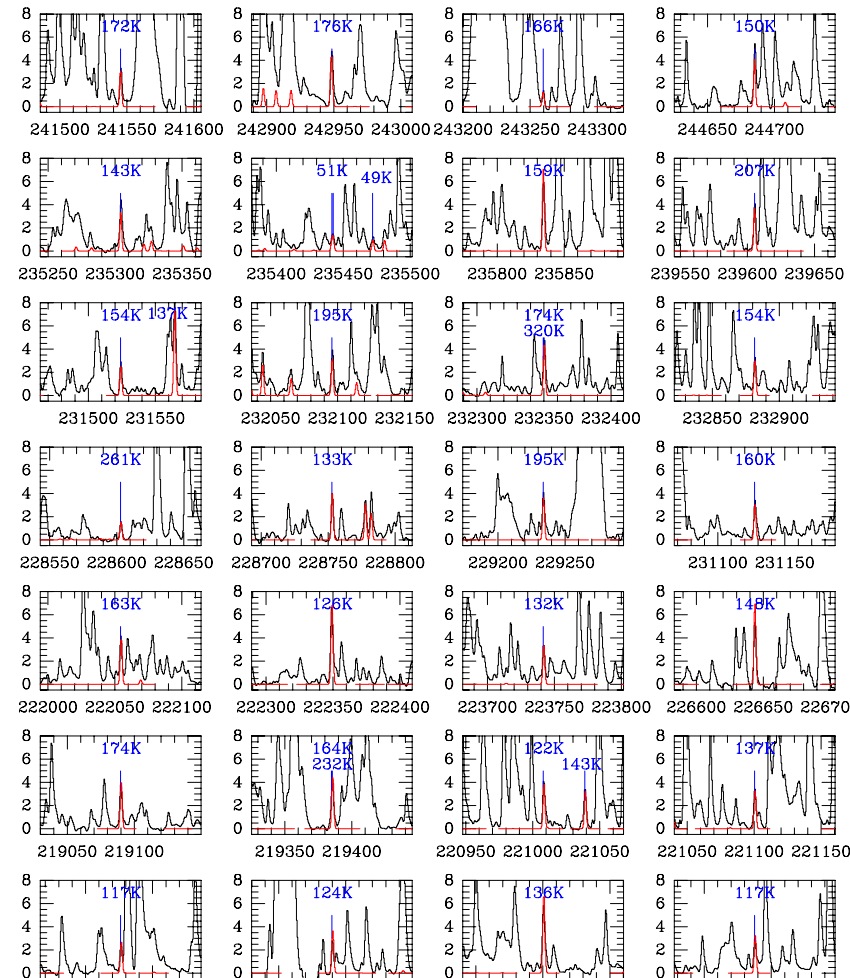

216800216850
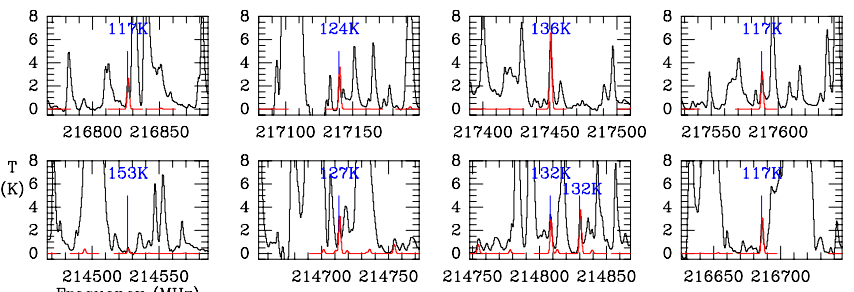

Fig. 7. ALMA spectrum (in black) towards the ethylene glycol peak at the ethylene glycol frequencies listed in Table 1 . The synthetic spectrum of ethylene glycol (EG) is overlaid in red and the value of the upper state energy $\left(E_{\mathrm{u}}\right)$ is indicated in blue.

with the ALMA observed spectrum at the $a G g^{\prime}$ peak leads to a maximum column density of $10^{15} \mathrm{~cm}^{-2}$, i.e. $\leq 1 / 5$ of the $a G g^{\prime}$ conformer. We have further assumed here that the partition functions of both conformers are identical since their rotational constants are very similar (Müller \& Christen 2004).

Because the $g G g^{\prime}$ conformer is $290 \mathrm{~K}$ higher in energy than $a G g^{\prime}$ and thus may probe hotter gas, we have also derived a 
synthetic spectrum at the $a G g^{\prime}$ peak for a temperature of $250 \mathrm{~K}$ significantly above the $145 \mathrm{~K}$ used in the previous section. We find a projected density upper limit of $2.3 \times 10^{15} \mathrm{~cm}^{-2}$. With this higher temperature, we have identified a few lines corresponding to $g G g^{\prime}$ transitions ${ }^{6}$ and with no apparent strong blending. However, the lines are too few and weak to dispel the confusion problem.

The tentative detection of ethylene glycol towards IRAS 16293-2422, source B, made by Jørgensen et al. (2012) corresponds to the $220249.8 \mathrm{MHz}$ transition of the $g G g^{\prime}$ conformer. The narrow lines detected towards the IRAS solar-type source B by Jorgensen et al. show little or no line confusion. The presence of ethylene glycol, however, must still await confirmation from detection of other transitions especially those from the most stable $a G g^{\prime}$ conformer. Unfortunately, the $220249.8 \mathrm{MHz} g G g^{\prime}$ transition is partially blended in our ALMA spectrum towards Orion-KL.

\section{Discussion}

\subsection{Comparison with interstellar sources and comets}

First, we summarize our findings in Orion-KL. The ethylene glycol peak is close to the prominent methyl formate position MF2 of Favre et al. (2011) and the derived temperature for ethylene glycol is similar to that derived for methyl formate. However, the column densities obtained for ethylene glycol, around $4.6 \times$ $10^{15} \mathrm{~cm}^{-2}$, are not very sensitive to the rotational temperature in this temperature range (see Fig. B.6 of Peng et al. 2012, in the case of methanol). Favre et al. (2011) find an upper limit for the glycolaldehyde column density of $3.5 \times 10^{14} \mathrm{~cm}^{-2}$ towards MF2 for a temperature of $140 \mathrm{~K}$ and a spatial resolution of 3.' $8 \times 2$.' 0 (line confusion does not allow us to get a significant upper limit at a higher spatial resolution). Peng et al. (2012) derive a column density of $4.2 \pm 0.4 \times 10^{18} \mathrm{~cm}^{-2}$ for the methanol peak named dM-1 (same position as MF2) with the same conditions. Using the ALMA data, we have also studied the ethanol emission (Despois et al., in prep.) and we find a column density of $\sim 2.5 \times 10^{16} \mathrm{~cm}^{-2}$ and a temperature of $\sim 140 \mathrm{~K}$ towards the ethanol peak of emission close to the ethylene glycol peak.

Clearly, and as in Sgr B2 (Belloche et al. 2013) and NGC 7129 FIRS 2 (Fuente et al. 2014), ethanol is more abundant than ethylene glycol (by a factor of 5 in our data). But ethylene glycol is at least 10 times more abundant than glycolaldehyde in Orion-KL whereas it is only twice more abundant in NGC 7129 FIRS 2 and both molecules have similar abundances towards Sgr B2. In all cases methanol is widespread and highly abundant.

Ethylene glycol is also more abundant than glycolaldehyde in comets and less abundant than methanol (by a factor 7-10, Crovisier et al. 2004b; Biver et al. 2014), whereas ethanol has not yet been detected with an upper limit 2.5 times lower than ethylene glycol.

Table 2 compares the column densities derived for ethylene glycol and related molecules towards Orion-KL, two other prominent interstellar sources and the 3 comets where ethylene glycol has been identified. We note that the comparison suffers

\footnotetext{
6 Frequencies of $g G g^{\prime}$ conformer transitions corresponding to not very blended lines in the ALMA spectrum at the $a G g^{\prime}$ peak: $220.2498 \mathrm{GHz}$, $224.8281 \mathrm{GHz}, 226.4111 \mathrm{GHz}, 227.2706 \mathrm{GHz}, 228.9154 \mathrm{GHz}$, $228.9179 \mathrm{GHz}, 228.9374 \mathrm{GHz}, 228.9399 \mathrm{GHz}, 229.4537 \mathrm{GHz}$, $229.4540 \mathrm{GHz}, 230.4622 \mathrm{GHz}, 232.7066 \mathrm{GHz}, 232.7076 \mathrm{GHz}$, $232.7080 \mathrm{GHz}, 241.6567 \mathrm{GHz}$.
}

from the fact that the spatial distribution is different for the different molecules and the spatial resolution is also different between the observed interstellar sources.

With the recent detection of ethylene glycol in two comets (Biver et al. 2014), whereas more abundant interstellar species like ethanol and glycolaldehyde are still undetected, the problem of ethylene glycol formation gets even more acute. Pathways to its formation are still under investigation. Walsh et al. (2014) summarize available grain-surface scenarios which include a) formation from $\mathrm{CH}_{2} \mathrm{OH}$ radical; b) hydrogenation of $\mathrm{OCCHO}$ itself resulting from atomic oxygen addition on $\mathrm{H}_{2} \mathrm{CO}$; c) some non detailed UV or CR processing of ices; d) possibility of thermal processing.

\subsection{Orion- $K L$}

Comparison of the ethylene glycol distribution in Orion-KL with other available physical or chemical tracers may shed some light on the molecular formation conditions in Orion-KL. We compared the maps of these tracers using the Aladin software ${ }^{7}$ (Bonnarel et al. 2000). This in turn could help us to understand the molecular complexity in other regions and perhaps in comets.

There is no obvious role played by temperature: the ethylene glycol peak does not show up in any of the high-resolution temperature maps of $\mathrm{CH}_{3} \mathrm{OH}$ (Beuther et al. 2006; Friedel \& Widicus Weaver 2012) and $\mathrm{NH}_{3}$ (Goddi et al. 2011a) or in any Mid-IR peaks (Okumura et al. 2011).

The various tracers do not show any concentration of matter at the ethylene glycol peak. On the contrary, high-resolution maps of ${ }^{13} \mathrm{CO}$ (Zapata et al. 2011) and continuum emission present a hole at the ethylene glycol peak and no clear association is seen with $\mathrm{NH}_{3}$ (Wilson et al. 2000).

At higher spatial resolution two continuum spots, C21 and C22 (Friedel \& Widicus Weaver 2011), appear on each side of the ethylene glycol peak. The northern spot is close to the SMA1 continuum source and coincides with a $22 \mathrm{GHz} \mathrm{H}_{2} \mathrm{O}$ maser (Gaume et al. 1998) and the MF6 methyl formate position of Favre et al. (2011). There is another $22 \mathrm{GHz}$ maser spot in the south at the same position as the MF2 methyl formate peak near but not exactly coincident with the continuum peak.

In the near-IR continuum as well as in $\mathrm{H}_{2} 2.2 \mu \mathrm{m}$ (Lacombe et al. 2004; Rouan, priv. comm.), the region appears as a dark patch; this is also the case at longer wavelength (e.g. at $20 \mu \mathrm{m}$, Robberto et al. 2005).

One short CO jet among jets identified by Zapata et al. (2009; and coming from the common "explosion centre" in between source I and BN) overlaps the ethylene glycol peak.

A precise comparison with ALMA maps of other oxygenated species is in preparation. According to previous studies by us and other groups, it appears clearly that the ethylene glycol distribution is definitely different from that of methyl formate and dimethyl ether (see e.g. Brouillet et al. 2013). It is also different from $\mathrm{CN}$ bearing species, such as $\mathrm{C}_{2} \mathrm{H}_{5} \mathrm{CN}$, from acetone $\mathrm{CH}_{3} \mathrm{COCH}_{3}$ (Peng et al. 2013; Friedel \& Snyder 2008; Widicus Weaver \& Friedel 2012), and from deuterated methanol (Peng et al. 2012). One of the ${ }^{13} \mathrm{C}$ methanol peaks in Peng et al. (2012) is however coincident with the ethylene glycol peak. Compared with HDO, which is typically released from grain surfaces, ethylene glycol appears to come from the same region as the main HDO emission delineated in the maps of Neill et al. (2013)

\footnotetext{
Centre de Données Astronomiques de Strasbourg, http: //aladin. u-strasbg. fr/aladin.gml
} 
Table 2. Comparison of the column densities and abundances of ethylene glycol and related molecules in interstellar sources and comets.

\begin{tabular}{|c|c|c|c|c|c|c|}
\hline \multirow[t]{2}{*}{ Molecule } & \multicolumn{3}{|c|}{$\begin{array}{l}\text { Column density } \\
\left(\mathrm{cm}^{-2}\right)\end{array}$} & \multicolumn{3}{|c|}{$\begin{array}{l}\text { Abundance }^{a} \\
\left(\% \mathrm{H}_{2} \mathrm{O}\right)\end{array}$} \\
\hline & Orion-KL & Sgr B2 & NGC 7129 & $\begin{array}{c}\text { C/1995 O1 } \\
\text { (Hale-Bopp) }\end{array}$ & $\begin{array}{l}\text { C/2012 F6 } \\
\text { (Lemmon) }\end{array}$ & $\begin{array}{c}\text { C/2013 A1 } \\
\text { (Lovejoy) }\end{array}$ \\
\hline$\left(\mathrm{CH}_{2} \mathrm{OH}\right)_{2}$ & $4.6 \times 10^{15}$ & $2.3 \times 10^{15}$ & $2.0 \times 10^{15}$ & 0.25 & 0.24 & 0.35 \\
\hline $\mathrm{CH}_{3} \mathrm{OH}$ & $4.2 \times 10^{18}$ & $5.6 \times 10^{18}$ & $3.4 \times 10^{20}$ & 2.4 & 1.6 & 2.6 \\
\hline $\mathrm{CH}_{3} \mathrm{CH}_{2} \mathrm{OH}$ & $2.5 \times 10^{16}$ & $2.1 \times 10^{16}$ & $3.0 \times 10^{18}$ & $\leq 0.1$ & $\leq 0.11$ & $\leq 0.14$ \\
\hline \multirow[t]{2}{*}{$\mathrm{CH}_{2} \mathrm{OHCHO}$} & $\leq 3.5 \times 10^{14}$ & $1.8 \times 10^{15}$ & $1.0 \times 10^{15}$ & $\leq 0.04$ & $\leq 0.08$ & $\leq 0.07$ \\
\hline & \multicolumn{6}{|c|}{ Relative abundance with respect to methanol } \\
\hline$\left(\mathrm{CH}_{2} \mathrm{OH}\right)_{2}$ & $1.1 \times 10^{-3}$ & $0.4 \times 10^{-3}$ & $0.006 \times 10^{-3}$ & 0.1 & 0.15 & 0.13 \\
\hline $\mathrm{CH}_{3} \mathrm{OH}$ & 1 & 1 & 1 & 1 & 1 & 1 \\
\hline $\mathrm{CH}_{3} \mathrm{CH}_{2} \mathrm{OH}$ & $6 \times 10^{-3}$ & $4 \times 10^{-3}$ & $9 \times 10^{-3}$ & $\leq 0.04$ & $\leq 0.07$ & $\leq 0.05$ \\
\hline $\mathrm{CH}_{2} \mathrm{OHCHO}$ & $\leq 0.08 \times 10^{-3}$ & $0.3 \times 10^{-3}$ & $0.003 \times 10^{-3}$ & $\leq 0.02$ & $\leq 0.05$ & $\leq 0.03$ \\
\hline
\end{tabular}

Notes. ${ }^{(a)}$ See Sect. 4.1 for references.

obtained from the ALMA-SV data. However, looking at the individual channel maps of the two molecules, we find at each velocity that the ethylene glycol emission peak is $\sim 1^{\prime \prime}$ west of the HDO peak.

These first comparisons of maps: a) seem to exclude that the temperature deduced from present-day observations could play a dominant role in the observed differences (but an earlier gas warming at ethylene glycol position is not excluded); and b) show that ethylene glycol is distinct from species like dimethyl ether and methyl formate, which are strong in the compact ridge and likely to be produced on grain mantles. The compact nature of the ethylene glycol emission compared to the more extended distributions of methanol and ethanol calls for a special production scenario. The above elements suggest that one possibility could be a collision of a high-velocity jet identified in $\mathrm{CO}$ (but possibly ionized) with a clump at the edge or in front of the main hot core condensation, which would be responsible for the ethylene glycol production and the hole in continuum emission. This collision could also be favourable to the excitation of the two $22 \mathrm{GHz}$ maser spots. Another scenario, involving UV photons and reactions with radicals, is not excluded. However, there is no special observational evidence in favour of this scenario, as no local source emitting strong UV emission has been identified yet in this area, and penetration of external UV photons into such a dense region would require voids in the dust distribution. The association of two $\mathrm{CH}_{2} \mathrm{OH}$ radicals to form $\left(\mathrm{CH}_{2} \mathrm{OH}\right)_{2}$, although appealing by its simplicity, is not favoured as the $\mathrm{CH}_{2} \mathrm{OH}$ radical has a low mobility due to $\mathrm{OH}$ bonds (Walsh et al. 2014).

\section{Continuum emission}

A map of the continuum emission at $1.3 \mathrm{~mm}$ in Orion-KL is a byproduct of our efforts to isolate the line emission by subtracting the underlying continuum. The ALMA data covers a total bandwidth of $32.9 \mathrm{GHz}$ centred at $230.171 \mathrm{GHz}$. For practical reasons, we have separated each one of the 20 spectral windows into four 1000-channel sub-windows, and we have searched for linefree channels in each sub-window using the following method.

If a channel map has no noticeable line emission, the difference between the maximum and the minimum intensities of the channel map will be lower than for channels with stronger molecular emission. Sorting out channels by increasing value of this difference, we have retained the best channel candidates to be line-free.
We then used the fact that if two channel maps are devoid (within the noise) of molecular emission, their difference is noise plus the difference in continuum emission. This contribution is negligible if the channel frequencies are close enough (as is the case in a sub-window). We selected the best of channel pairs with a low and spatially uniform noise difference.

Next, we eliminated channel pairs whose frequencies are closer than a typical line width, and might thus have identical molecular emission, inappropriately passing our second criterion.

Then we obtained the continuum map for a given subwindow by averaging the two channel maps of the best pair.

Finally, we checked the coherence of the continuum maps obtained for adjacent sub-windows.

It was not possible to identify line-free channels for all the sub-windows, in that case, we took the continuum emission from a sub-window adjacent in frequency. We have thus obtained an 80-channel continuum cube in the range $213.7 \mathrm{GHz}$ to $246.6 \mathrm{GHz}$.

Figure 8 compares the continuum maps obtained at the same frequency and with a similar spatial resolution with ALMA (this work) and the IRAM Plateau de Bure Interferometer (Favre et al. 2011). There is a good agreement between both maps which show the same four main components. The ALMA hot core position is $\left(\alpha_{\mathrm{J} 2000}=05^{\mathrm{h}} 35^{\mathrm{m}} 14^{\mathrm{s}} .55, \delta_{\mathrm{J} 2000}=-05^{\circ} 22^{\prime} 31^{\prime \prime} .3\right)$ and it corresponds, within $0.2^{\prime \prime}$, to the Ca continuum peak identified in Favre et al. (2011). The slight differences observed in the ALMA and IRAM interferometer maps are likely due to different spatial filtering of the two arrays.

Figure 9 shows the continuum variation with respect to the frequency over the whole ALMA-SV bandwidth. The spectrum is flat at the position of the ethylene glycol peak whereas it rises with frequency at the hot core position. This seems to be related to the sizes of the different continuum sub-sources associated with our main continuum component. In particular, if the continuum sub-source close to the hot core position is more compact than the synthesized beam, its intensity will vary as the beam size varies across the frequency range covered by the ALMASV data. We further note that our unresolved main continuum component (hot core) shows a NE-SW extension which encompasses the two sub-sources seen in the sub-arcsec $870 \mu \mathrm{m}$ dust continuum map of Tang et al. (2010) and the complex emission recently mapped with $0.5^{\prime \prime}$ ALMA resolution by Hirota et al. (2015). 

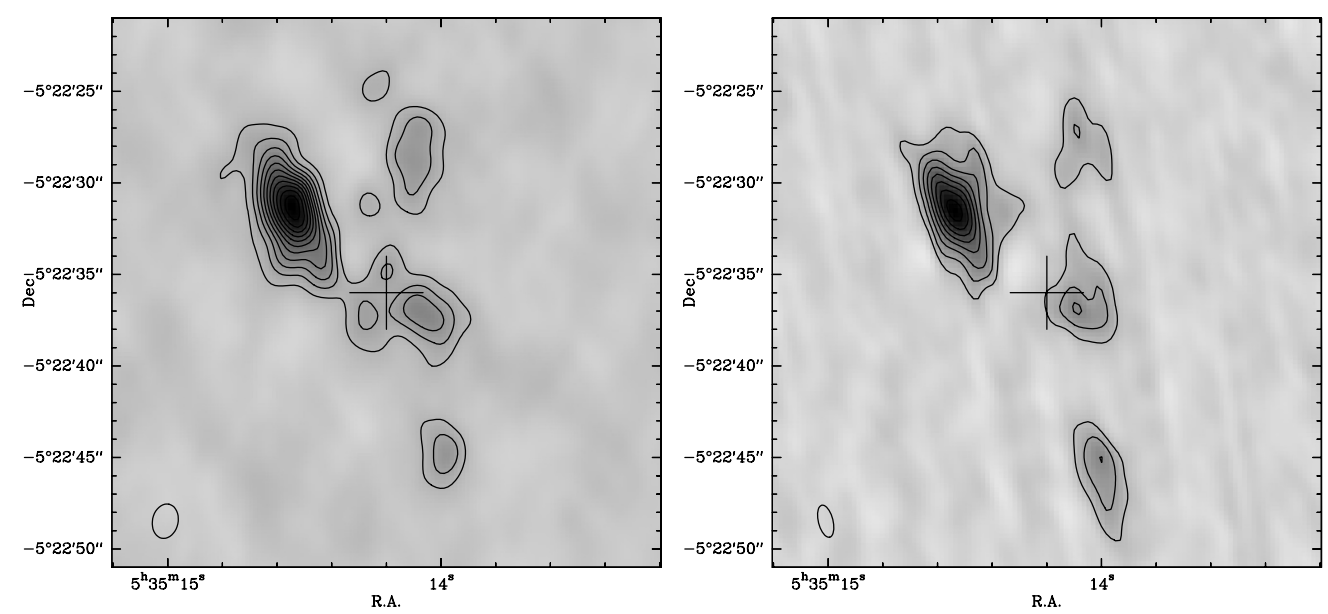

Fig. 8. Left: continuum map obtained with ALMA at $223 \mathrm{GHz}$. The beam shown in the bottom left corner is $1.92^{\prime \prime} \times 1.38^{\prime \prime}$. Right: continuum map obtained with the IRAM Plateau de Bure Interferometer at $223 \mathrm{GHz}$ (Favre et al. 2011). The beam shown in the bottom left corner is $1.79^{\prime \prime} \times$ $0.79^{\prime \prime}$. The level step and first contour for the two maps are $1 \mathrm{~K}$.

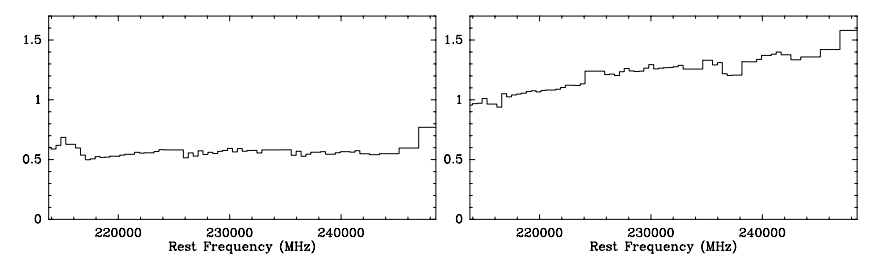

Fig. 9. Flux density per beam (in Jy/beam) versus frequency across ALMA band 6. Left: at the position of the ethylene glycol peak. Right: at the hot core position.

\section{Conclusions}

1. We detected the $a G g^{\prime}$ conformer of ethylene glycol in Orion KL. The emission peaks towards the hot core close to the main continuum peak, about $2^{\prime \prime}$ to the southwest. The distribution is compact with a deconvolved size $\leq 2.4^{\prime \prime} \times 1.1^{\prime \prime}$. It is clearly different from that of other complex O-bearing species like methyl formate, dimethyl ether, acetone, and ethanol.

2. We deduced a beam averaged column density $N_{a G g^{\prime}}$ of $4.6 \pm$ $0.8 \times 10^{15} \mathrm{~cm}^{-2}$ and a rotational temperature $T$ of $145 \pm 30 \mathrm{~K}$ from a population diagram. Using the continuum map, we find an ethylene glycol abundance with respect to $\mathrm{H}_{2}$ of the order of $10^{-9}$.

3. Taking methanol $\mathrm{CH}_{3} \mathrm{OH}$ as a reference, the relative abundance of ethylene glycol is of the order of $10^{-3}$ in Orion-KL, similar to that in Sgr B2. We confirm with Orion-KL that the ethylene glycol abundance in interstellar sources is much lower than in the three comets where it has been detected with a ratio in the range 0.1-0.15. This result places ethylene glycol clearly outside the correlation found by Bockelée-Morvan et al. (2000) between Comet C/1995 O1 (Hale-Bopp) and four well-studied compact sources, including Orion-KL.

4. The simple alcohol ethanol $\mathrm{CH}_{3} \mathrm{CH}_{2} \mathrm{OH}$ is more abundant than the di-alcohol $\mathrm{CH}_{2} \mathrm{OHCH}_{2} \mathrm{OH}$ by a factor of 5 in Orion-KL and even larger factors were found in Sgr B2 and NGC 7129. This result confirms a marked difference with the comets where, on the opposite, an upper limit of 0.5 has been derived for the ethanol/ethylene glycol ratio.

5. We only identified weak lines of the $g G g^{\prime}$ conformer at the expected frequencies, and all strong lines were blended. Hence we conclude that the $g G g^{\prime}$ conformer is not detected in our study, and we derive a column density less than 0.2 times the $a G g^{\prime}$ conformer's at the emission peak of the latter.

6. The identification of the ethylene glycol $a G g^{\prime}$ conformer was made possible thanks to the ALMA-SV data. A large frequency range is necessary to identify complex molecules and interferometric maps of the transitions considerably helped us in diminishing the line confusion problem.

7. Molecules have different spatial distribution even when a priori of a same group (e.g. large O-bearing molecules). This should be taken into account in future comparisons between interstellar sources observed at different spatial resolution as well as between interstellar sources and comets. It might bring diversity in forming planets and their content in prebiotic species.

Acknowledgements. We thank the referee for his help in improving the paper. This paper makes use of the following ALMA data: ADS/JAO.ALMA\#2011.0.00009.SV. ALMA is a partnership of ESO (representing its member states), NSF (USA) and NINS (Japan), together with NRC (Canada) and NSC and ASIAA (Taiwan), in cooperation with the Republic of Chile. The Joint ALMA Observatory is operated by ESO, AUI/NRAO and NAOJ. We used for the analysis the CASSIS software developed by IRAP-UPS/CNRS (http://cassis.irap.omp.eu). We thank the IRAM staff in Grenoble and, in particular, Jan-Martin Winter. This study started with IRAM PdBI data kindly provided by T. Jacq. M. Ali Dib's internship was of great help in the first developments and tests of the continuum subtraction method. This work was supported by CNRS national programs PCMI (Physics and Chemistry of the Interstellar Medium) and GDR Exobiology.

\section{References}

Belloche, A., Müller, H. S. P., Menten, K. M., Schilke, P., \& Comito, C. 2013 , A\&A, 559, A47

Beuther, H., Zhang, Q., Reid, M. J., et al. 2006, ApJ, 636, 323

Biver, N., Bockelée-Morvan, D., Debout, V., et al. 2014, A\&A, 566, L5

Bockelée-Morvan, D., Lis, D. C., Wink, J. E., et al. 2000, A\&A, 353, 1101

Bockelée-Morvan, D., Gautier, D., Hersant, F., Huré, J.-M., \& Robert, F. 2002, A\&A, 384, 1107

Bonnarel, F., Fernique, P., Bienaymé, O., et al. 2000, A\&AS, 143, 33

Brouillet, N., Despois, D., Baudry, A., et al. 2013, A\&A, 550, A46

Ceccarelli, C., Caselli, P., Bockelee-Morvan, D., et al. 2014, in Protostars and

Planets VI, eds. H. Beuther, et al. (Tucson: University of Arizona Press), 859 Christen, D., \& Müller, H. S. P. 2003, Phys. Chem. Chem. Phys., 5, 3600

Christen, D., Coudert, L. H., Suenram, R. D., \& Lovas, F. J. 1995, J. Mol. Spectr., 172,57

Christen, D., Coudert, L. H., Larsson, J. A., \& Cremer, D. 2001, J. Mol. Spectr., 205, 185

Clark, B. G. 1980, A\&A, 89, 377 
Crovisier, J., Leech, K., Bockelee-Morvan, D., et al. 1997, Science, 275, 1904 Crovisier, J., Bockelée-Morvan, D., Biver, N., et al. 2004a, A\&A, 418, L35 Crovisier, J., Bockelée-Morvan, D., Colom, P., et al. 2004b, A\&A, 418, 1141

Favre, C., Despois, D., Brouillet, N., et al. 2011, A\&A, 532, A32

Friedel, D. N., \& Snyder, L. E. 2008, ApJ, 672, 962

Friedel, D. N., \& Widicus Weaver, S. L. 2011, ApJ, 742, 64

Friedel, D. N., \& Widicus Weaver, S. L. 2012, ApJS, 201, 17

Friedel, D. N., Snyder, L. E., Turner, B. E., \& Remijan, A. 2004, ApJ, 600, 234

Fuente, A., Cernicharo, J., Caselli, P., et al. 2014, A\&A, 568, A65

Gaume, R. A., Wilson, T. L., Vrba, F. J., Johnston, K. J., \& Schmid-Burgk, J. 1998, ApJ, 493, 940

Goddi, C., Greenhill, L. J., Humphreys, E. M. L., Chandler, C. J., \& Matthews, L. D. 2011a, ApJ, 739, L13

Goddi, C., Humphreys, E. M. L., Greenhill, L. J., Chandler, C. J., \& Matthews, L. D. 2011 b, ApJ, 728, 15

Goldsmith, P. F., \& Langer, W. D. 1999, ApJ, 517, 209

Guélin, M., Brouillet, N., Cernicharo, J., Combes, F., \& Wooten, A. 2008, Ap\&SS, 313, 45

Halfen, D. T., Apponi, A. J., Woolf, N., Polt, R., \& Ziurys, L. M. 2006, ApJ, 639, 237

Herbst, E., \& van Dishoeck, E. F. 2009, ARA\&A, 47, 427

Hirota, T., Kim, M. K., Kurono, Y., \& Honma, M. 2015, ApJ, in press

Hollis, J. M., Lovas, F. J., Jewell, P. R., \& Coudert, L. H. 2002, ApJ, 571, L59

Irvine, W. M., Schloerb, F. P., Crovisier, J., Fegley, Jr., B., \& Mumma, M. J. 2000, in Protostars and Planets IV, 1159

Jørgensen, J. K., Favre, C., Bisschop, S. E., et al. 2012, ApJ, 757, L4

Lacombe, F., Gendron, E., Rouan, D., et al. 2004, A\&A, 417, L5
Maury, A. J., Belloche, A., André, P., et al. 2014, A\&A, 563, L2 Müller, H. S. P., \& Christen, D. 2004, J. Mol. Spectr., 228, 298

Müller, H. S. P., Thorwirth, S., Roth, D. A., \& Winnewisser, G. 2001, A\&A, 370, L49

Müller, H. S. P., Schlöder, F., Stutzki, J., \& Winnewisser, G. 2005, J. Mol. Struct., 742,215

Mumma, M. J., \& Charnley, S. B. 2011, ARA\&A, 49, 471

Neill, J. L., Wang, S., Bergin, E. A., et al. 2013, ApJ, 770, 142

Nummelin, A., Bergman, P., Hjalmarson, A., et al. 2000, ApJS, 128, 213

Okumura, S.-I., Yamashita, T., Sako, S., et al. 2011, PASJ, 63, 823

Peng, T.-C., Despois, D., Brouillet, N., Parise, B., \& Baudry, A. 2012, A\&A, 543, A152

Peng, T.-C., Despois, D., Brouillet, N., et al. 2013, A\&A, 554, A78

Requena-Torres, M. A., Martín-Pintado, J., Martín, S., \& Morris, M. R. 2008, ApJ, 672, 352

Robberto, M., Beckwith, S. V. W., Panagia, N., et al. 2005, AJ, 129, 1534

Snyder, L. E., Lovas, F. J., Hollis, J. M., et al. 2005, ApJ, 619, 914

Tang, Y., Ho, P. T. P., Koch, P. M., \& Rao, R. 2010, ApJ, 717, 1262

Tercero, B., Cernicharo, J., Pardo, J. R., \& Goicoechea, J. R. 2010, A\&A, 517, A96

Walsh, C., Millar, T. J., Nomura, H., et al. 2014, A\&A, 563, A33

Widicus Weaver, S. L., \& Friedel, D. N. 2012, ApJS, 201, 16

Wilson, T. L., Gaume, R. A., Gensheimer, P., \& Johnston, K. J. 2000, ApJ, 538, 665

Wright, M. C. H., Plambeck, R. L., \& Wilner, D. J. 1996, ApJ, 469, 216

Zapata, L. A., Schmid-Burgk, J., Ho, P. T. P., Rodríguez, L. F., \& Menten, K. M. 2009, ApJ, 704, L45

Zapata, L. A., Loinard, L., Schmid-Burgk, J., et al. 2011, ApJ, 726, L12

Pages 11 to 18 are available in the electronic edition of the journal at http://www . aanda. org 


\section{Appendix A: ALMA spectrum towards the ethylene glycol peak}
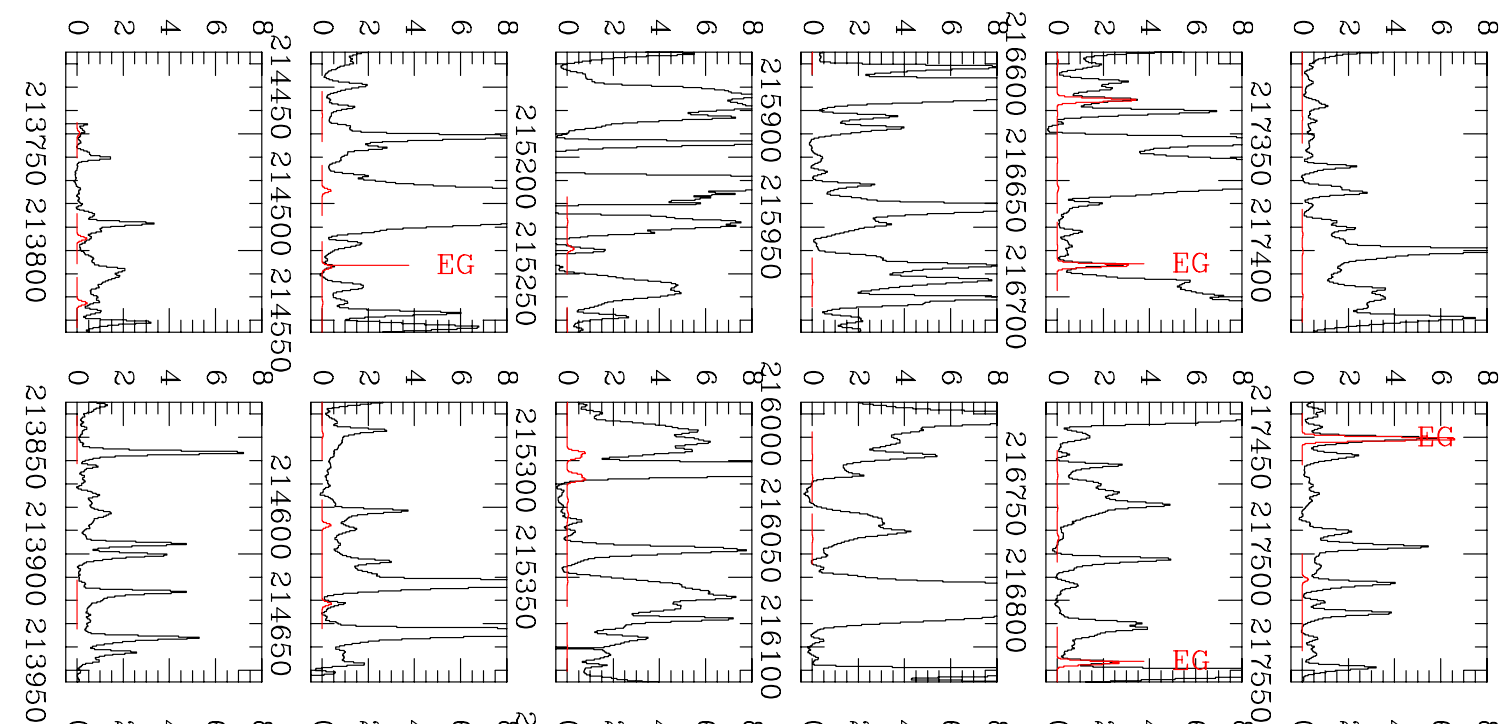

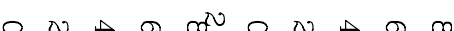
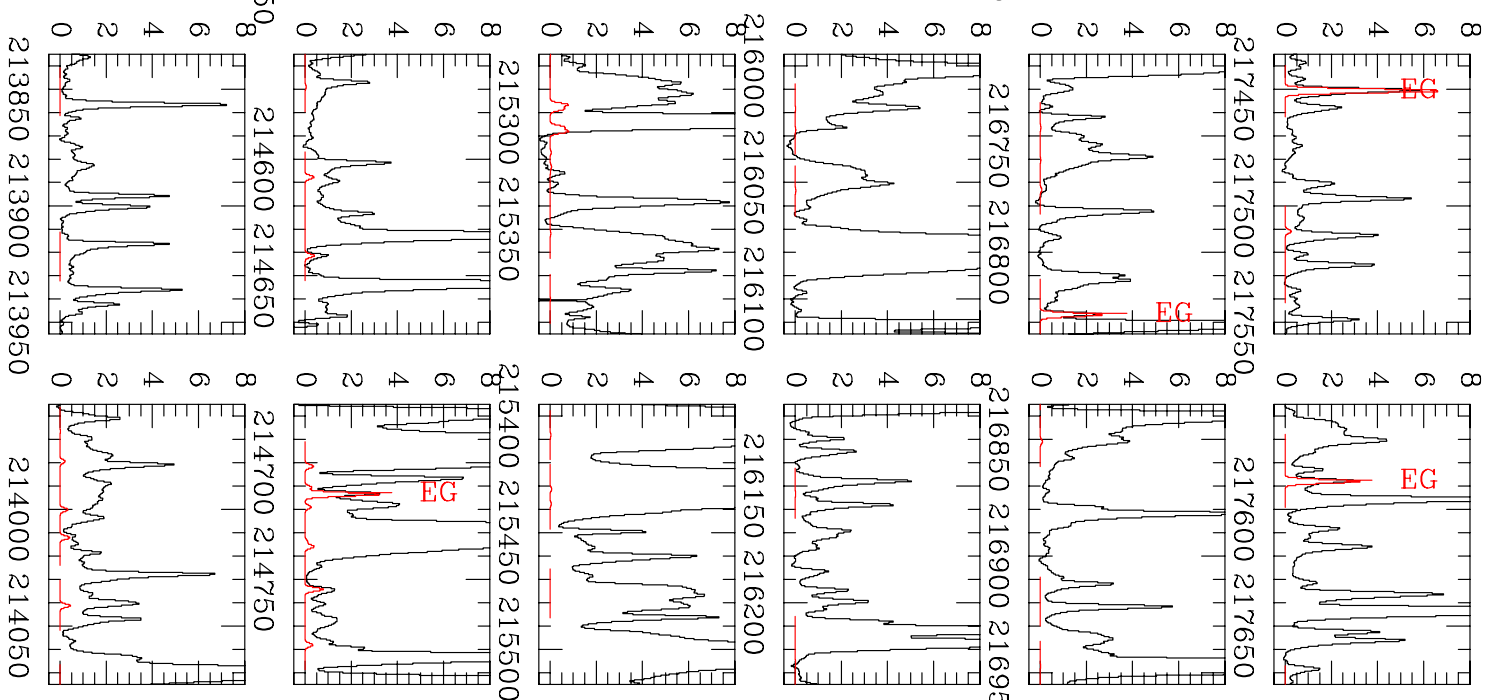

O v A a
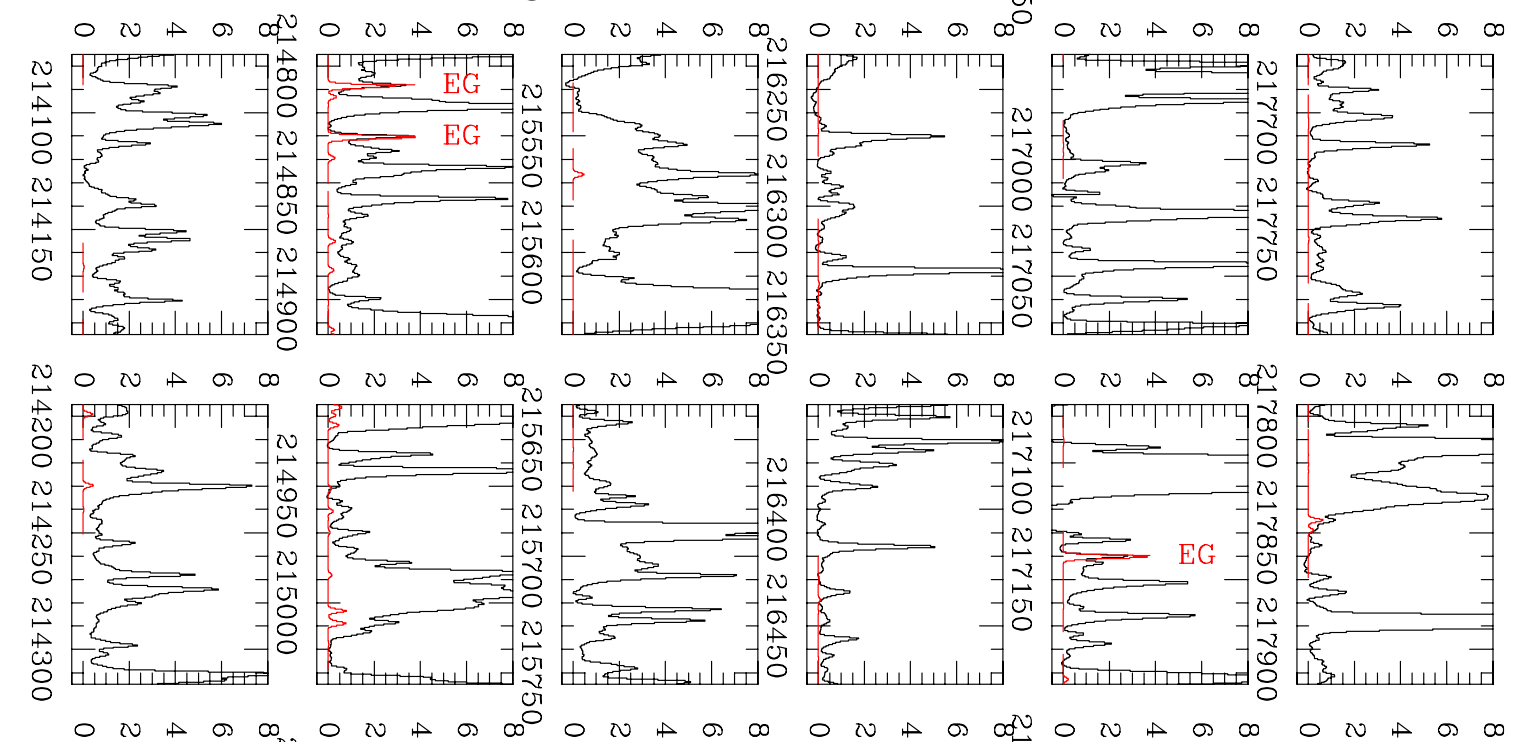

o va a $\infty$ a d a a
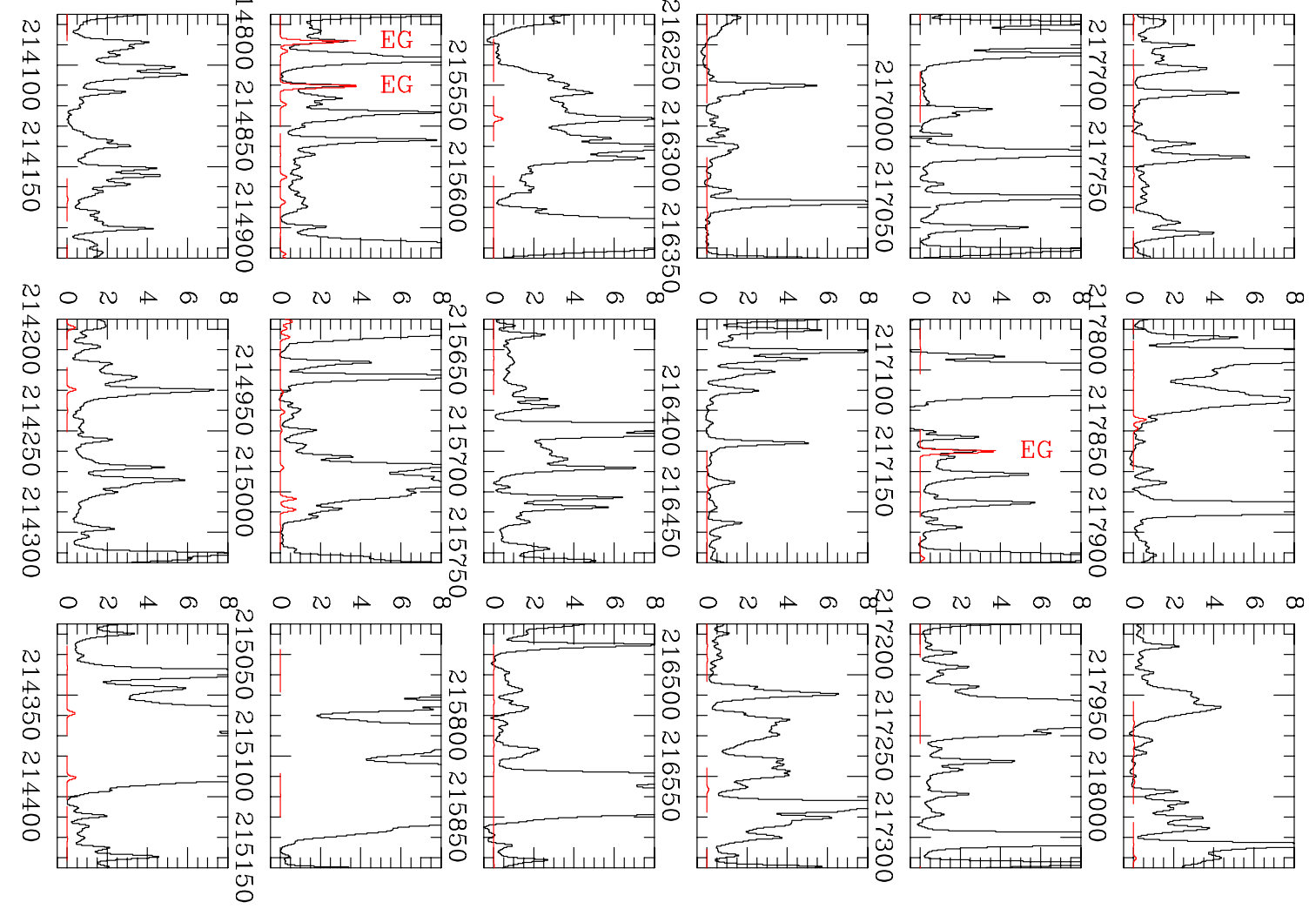

Fig. A.1. ALMA spectrum (in black) towards the ethylene glycol peak. The synthetic spectrum is overlaid in red. The parameters used for the synthetic spectrum are: $v_{\mathrm{LSR}}=7.6 \mathrm{~km} \mathrm{~s}^{-1}, \Delta v_{1 / 2}=2.3 \mathrm{~km} \mathrm{~s}^{-1}, N_{a G q^{\prime}}=4.6 \times 10^{15} \mathrm{~cm}^{-2}, T=145 \mathrm{~K}$. Each box corresponds to a $120 \mathrm{MHz}$ bandwidth and the ordinates are the intensities in $\mathrm{K}$. The frequencies (in $\mathrm{MHz}$ ) increase from left to right and from bottom to top. The transitions listed in Table 1 are indicated on the plots by "EG". 

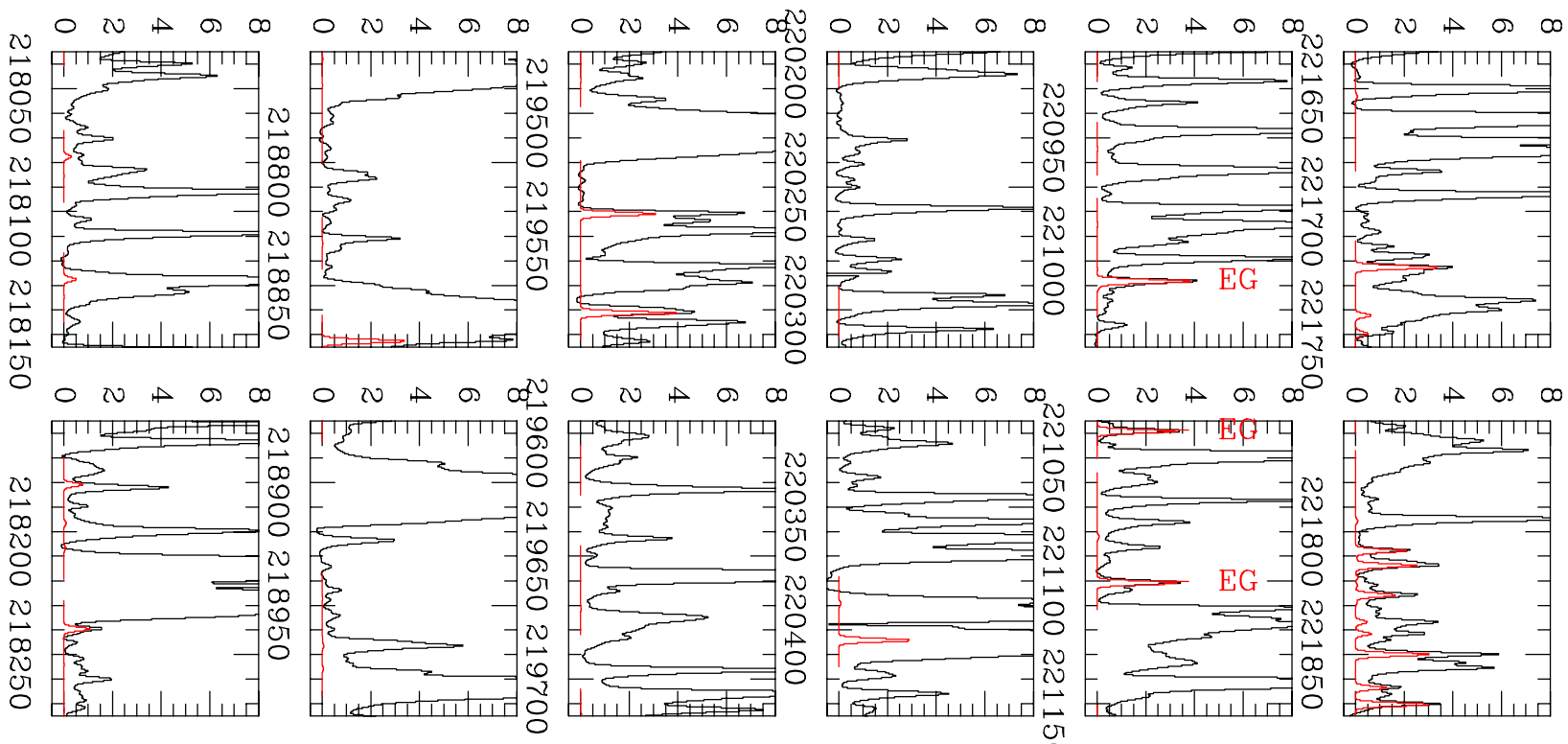

O N A a O O O N A
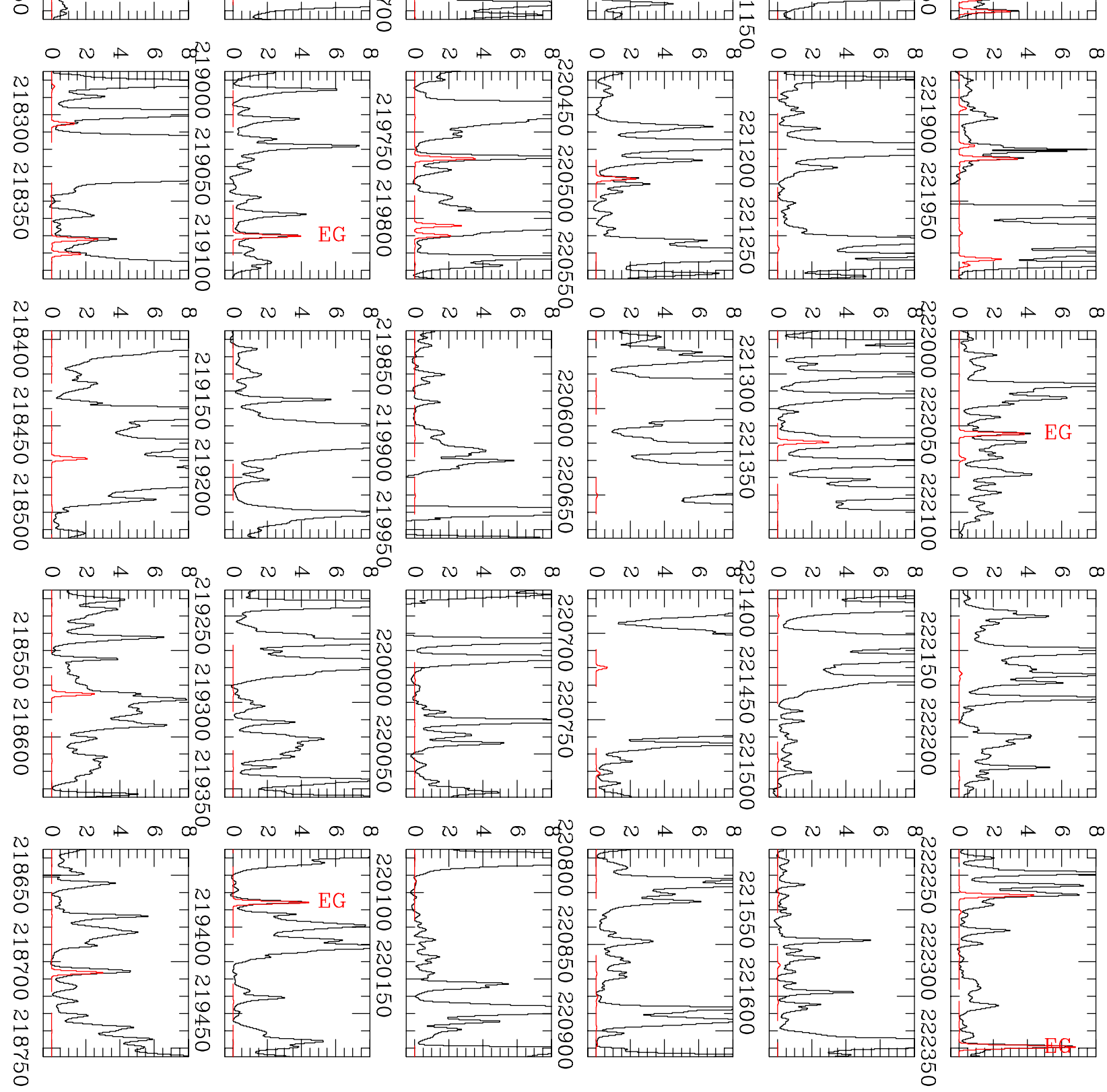

Fig. A.1. continued. 
N. Brouillet et al.: Antifreeze in the hot core of Orion
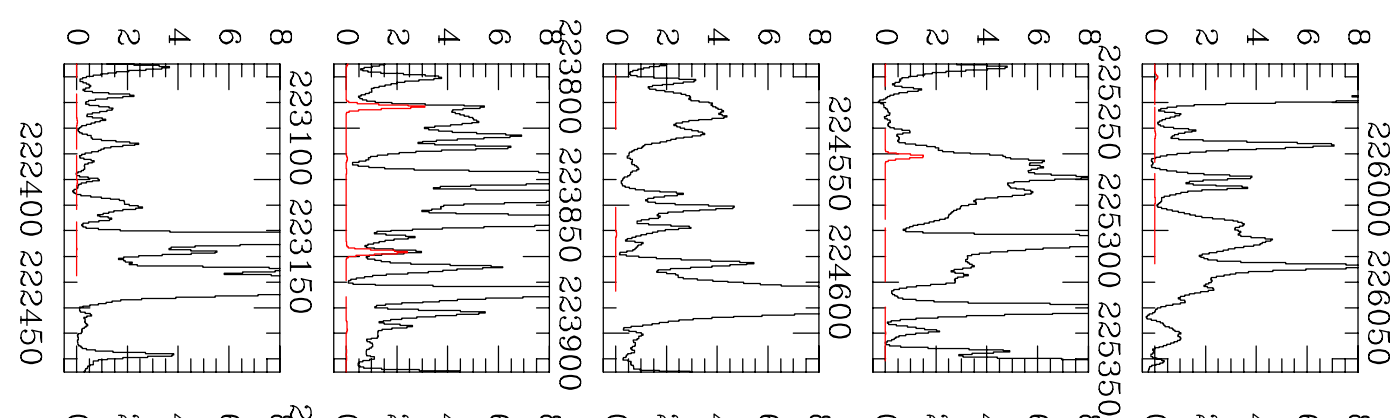

- vis a $\infty$

- v
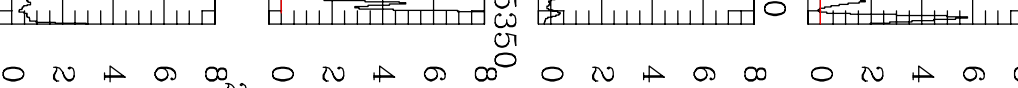

v
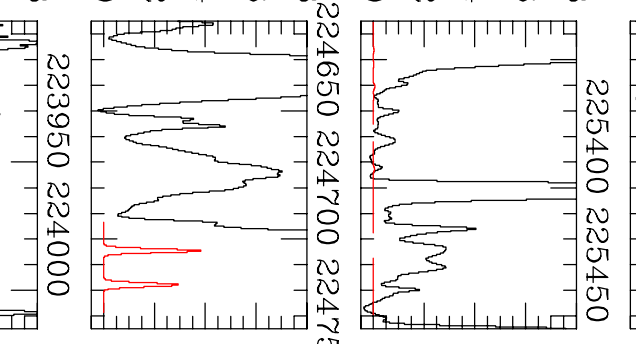

o va a $\infty$ a v
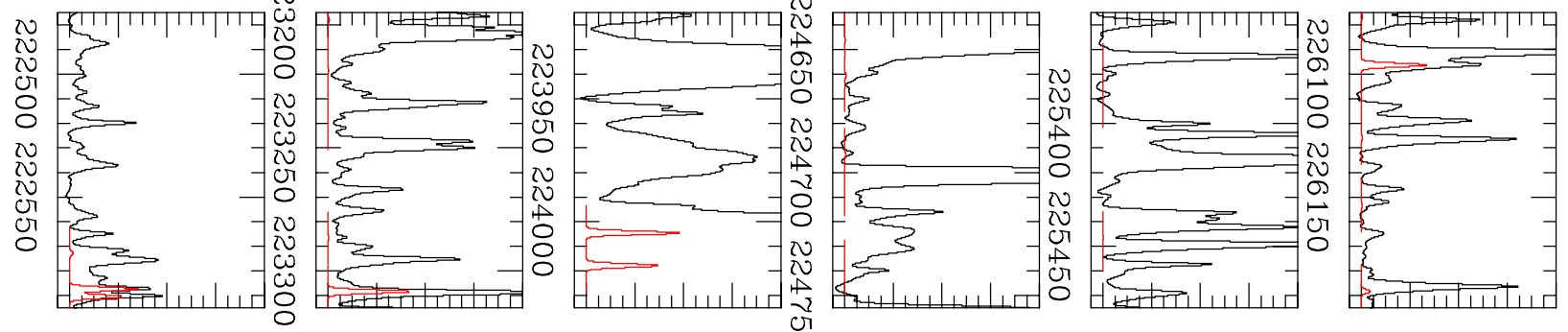

N 0 U

o va a o

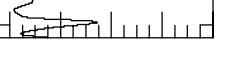
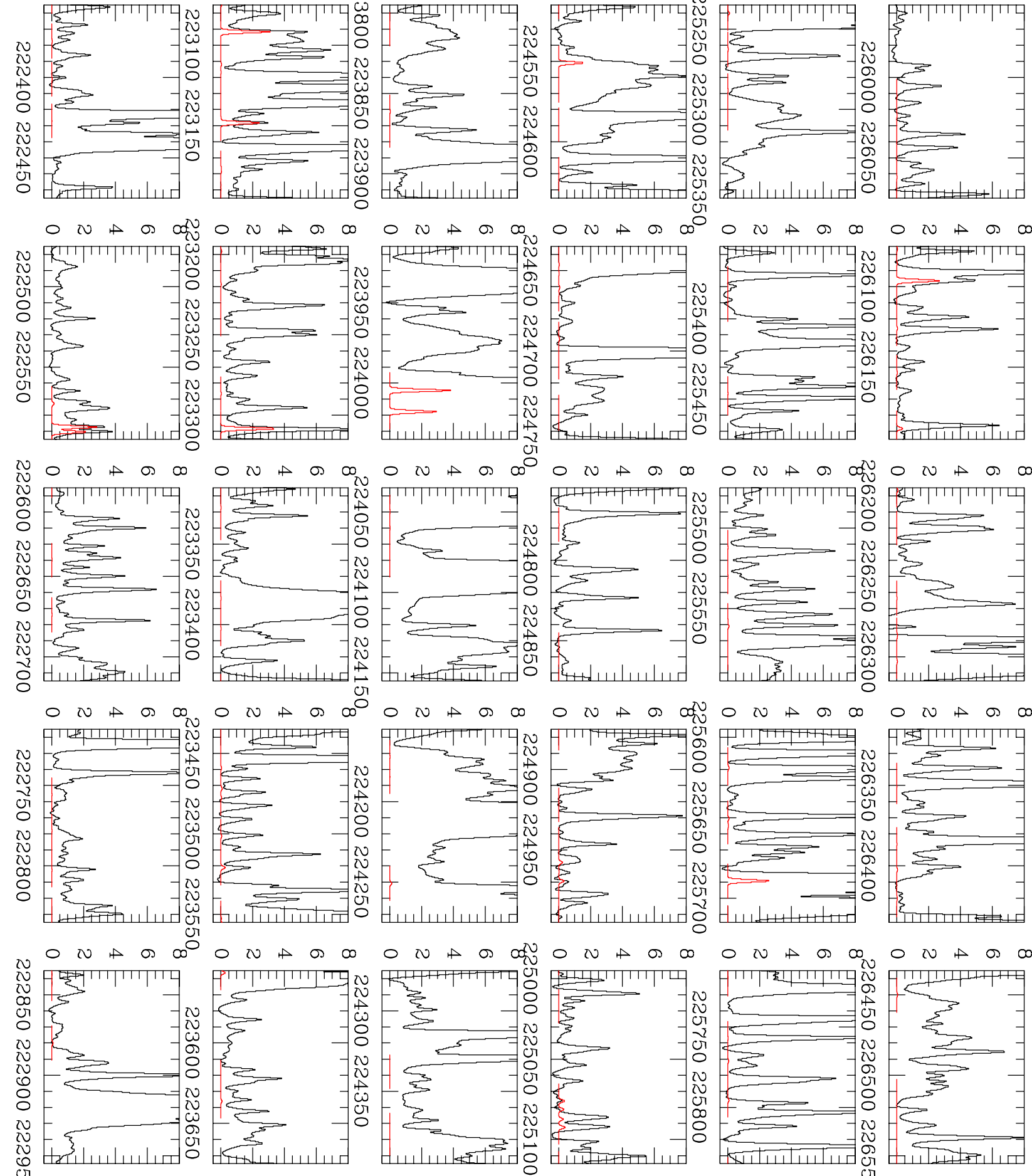

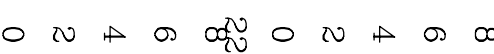
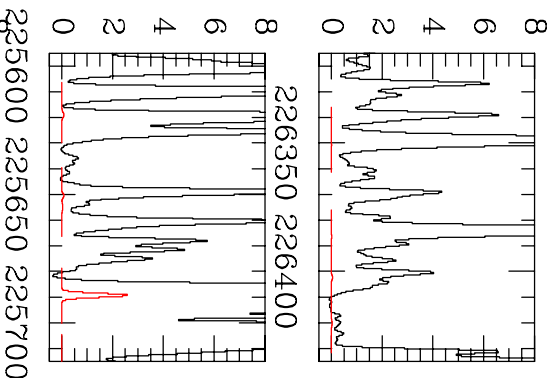
or

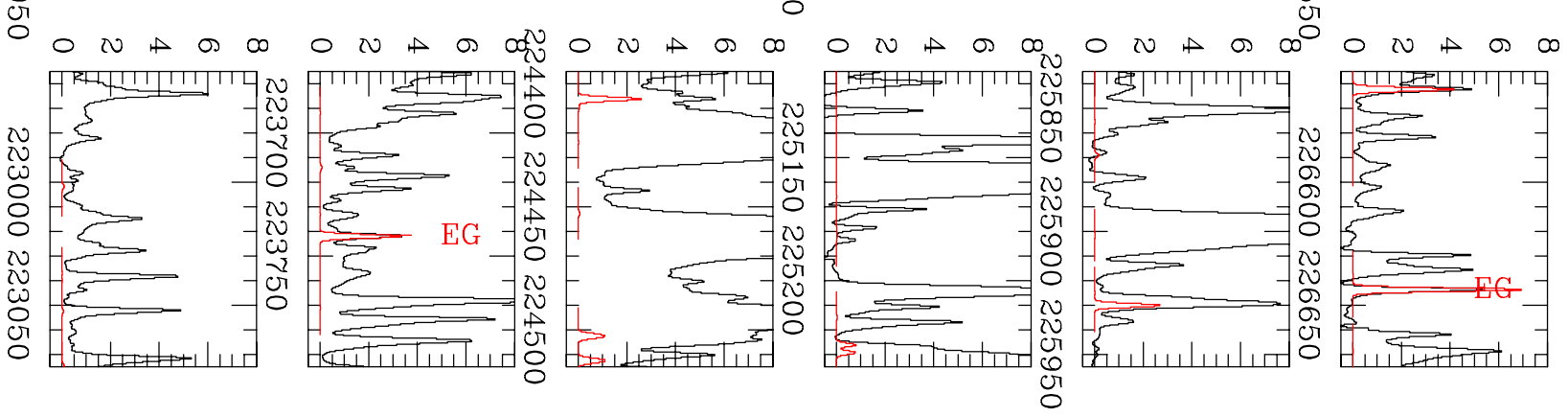

Fig. A.1. continued. 
o va a an o n a a

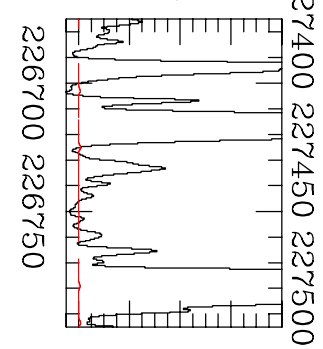

No n

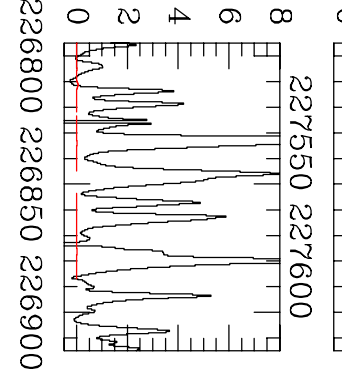

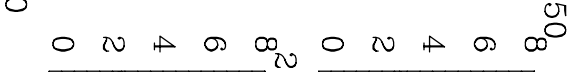
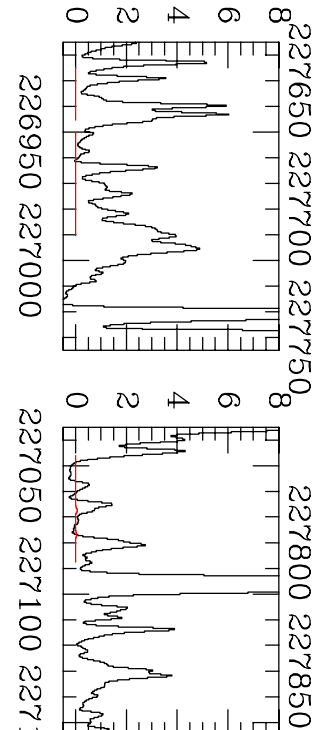

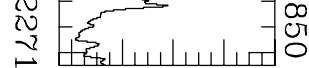

宁
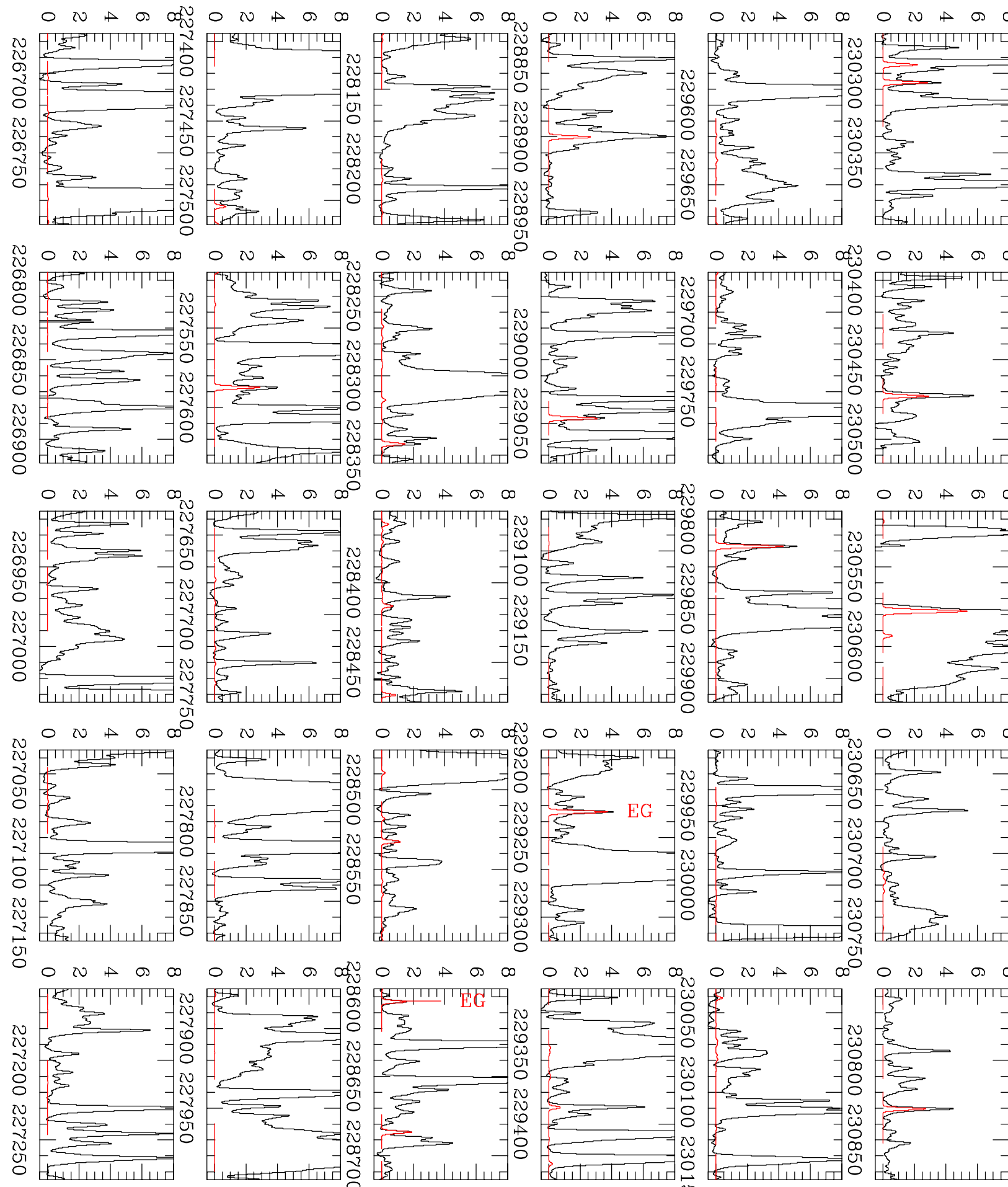

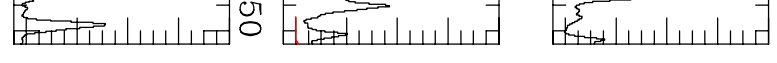

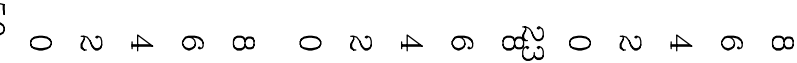
- va a $\infty_{n} 0$ na a
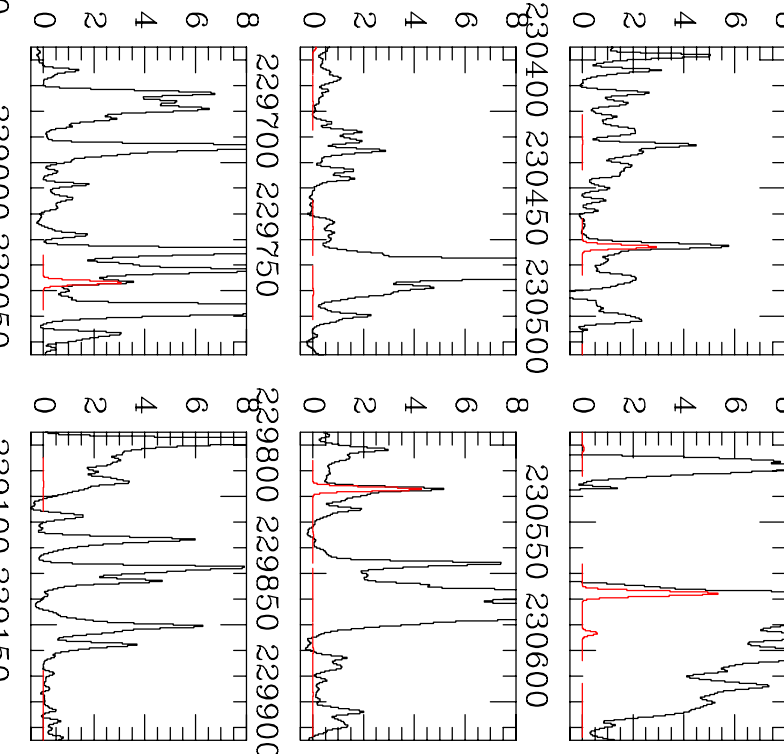

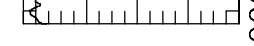
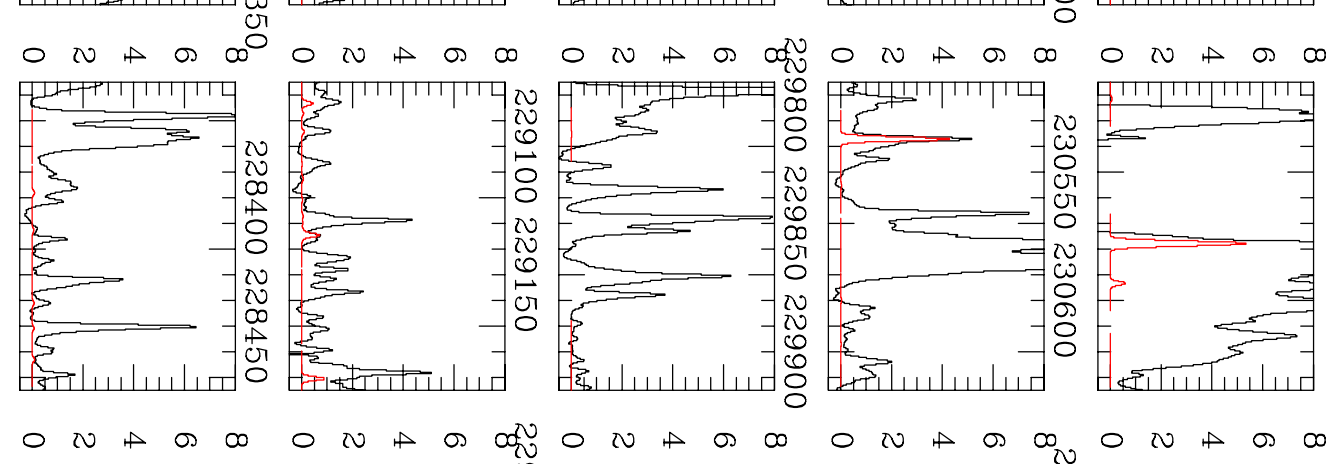

ov 0 a d o v
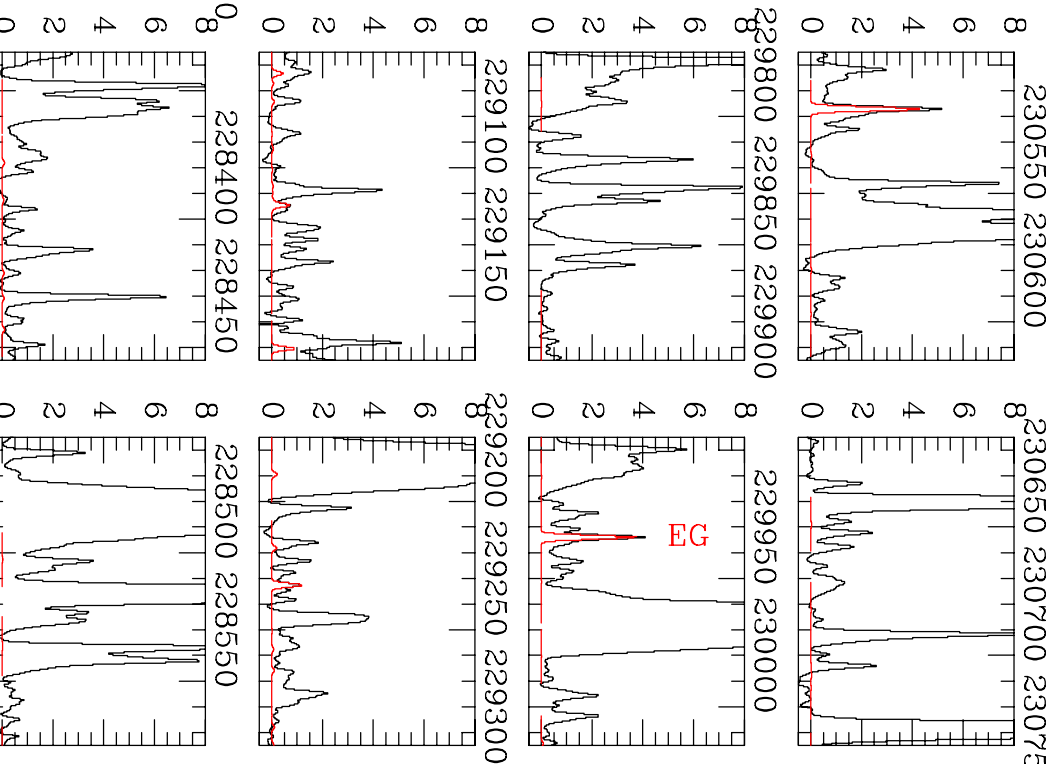

- N
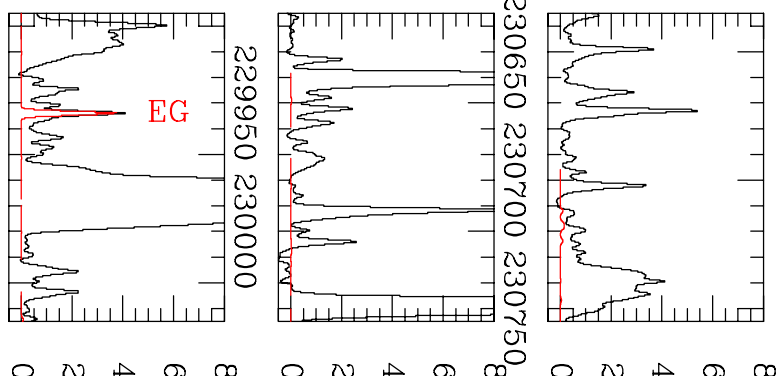

जो
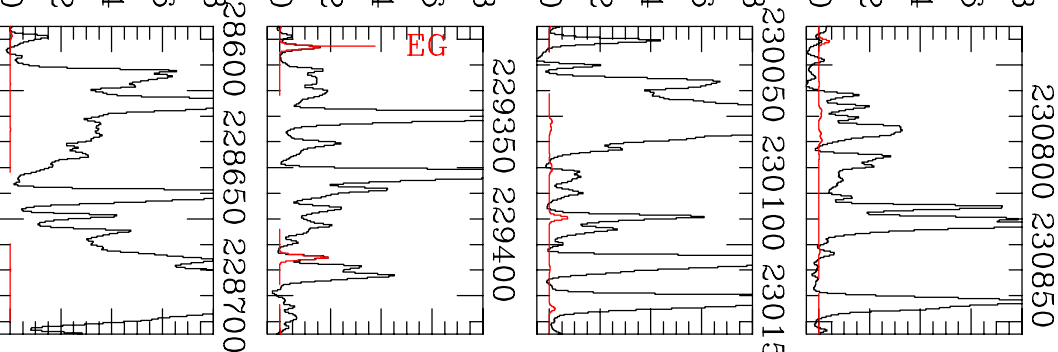

- v a o $\infty$

- v
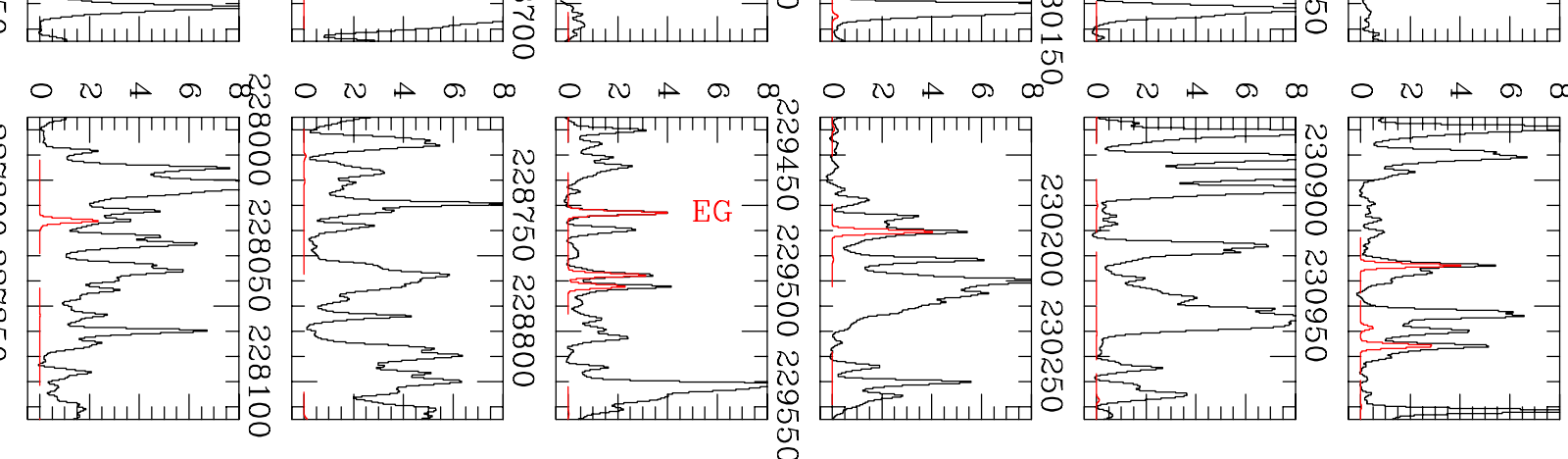

O

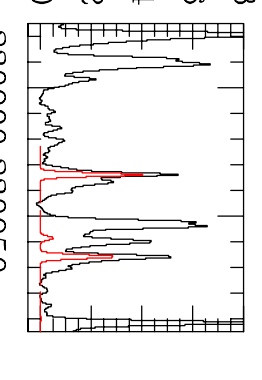

Fig. A.1. continued. 
N. Brouillet et al.: Antifreeze in the hot core of Orion
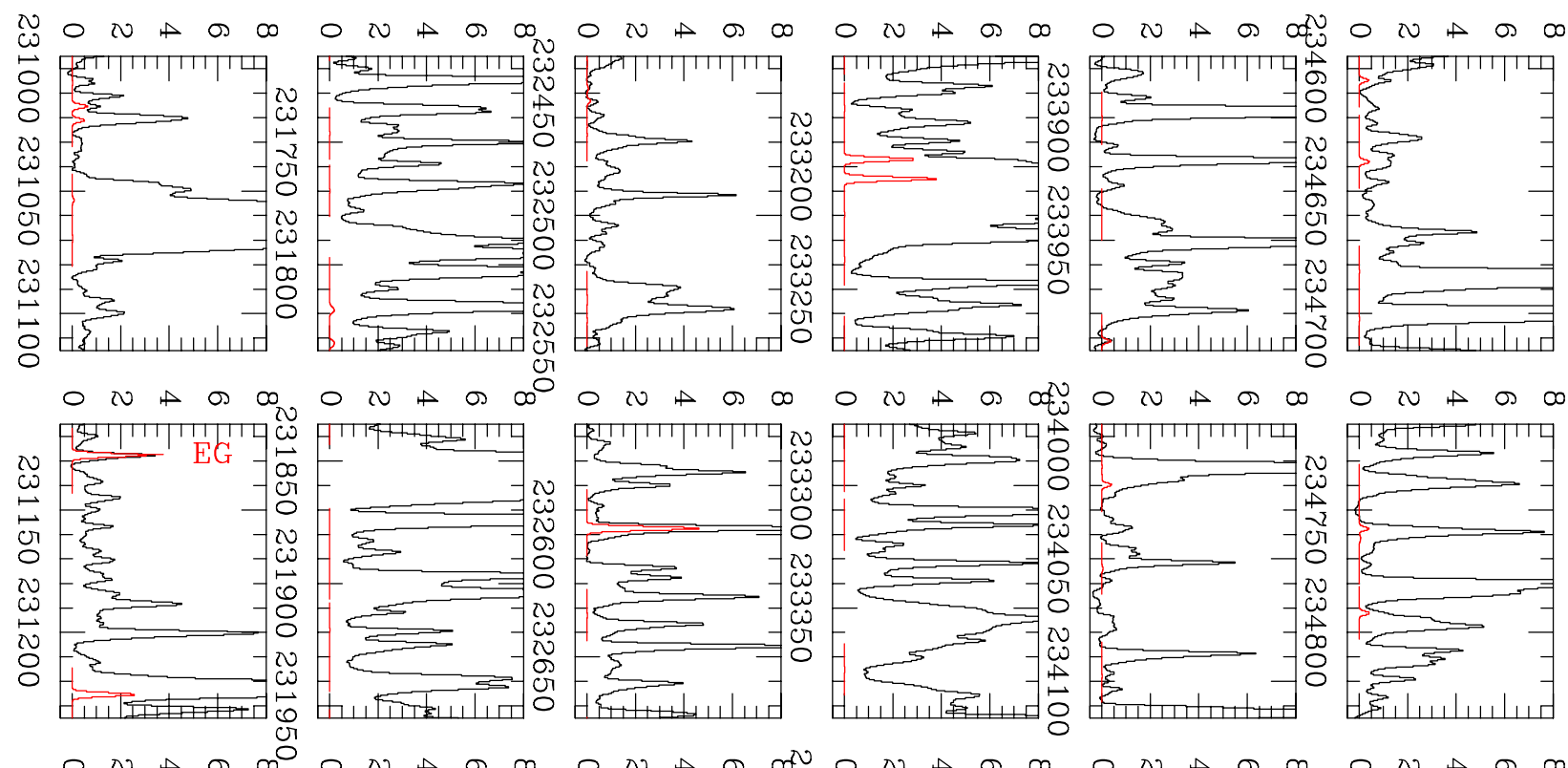

o

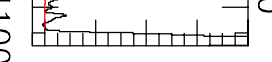

لملسلسلس
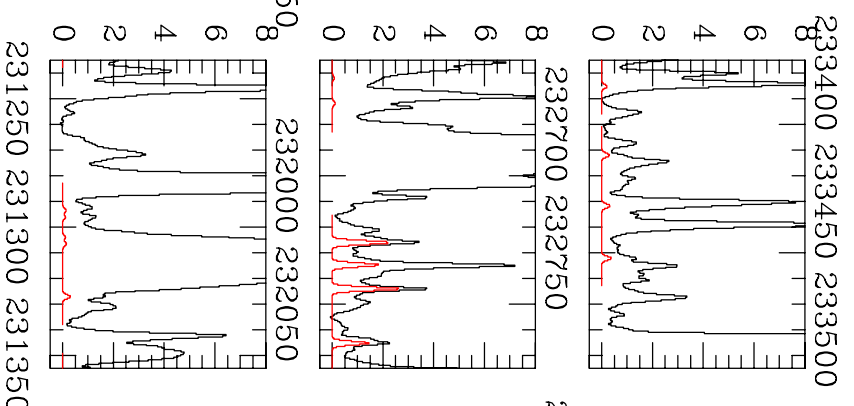

O O N
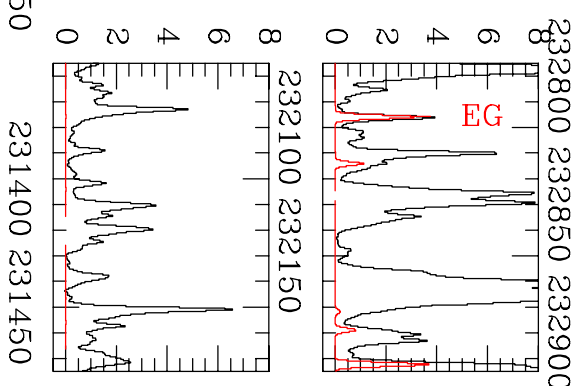

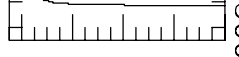
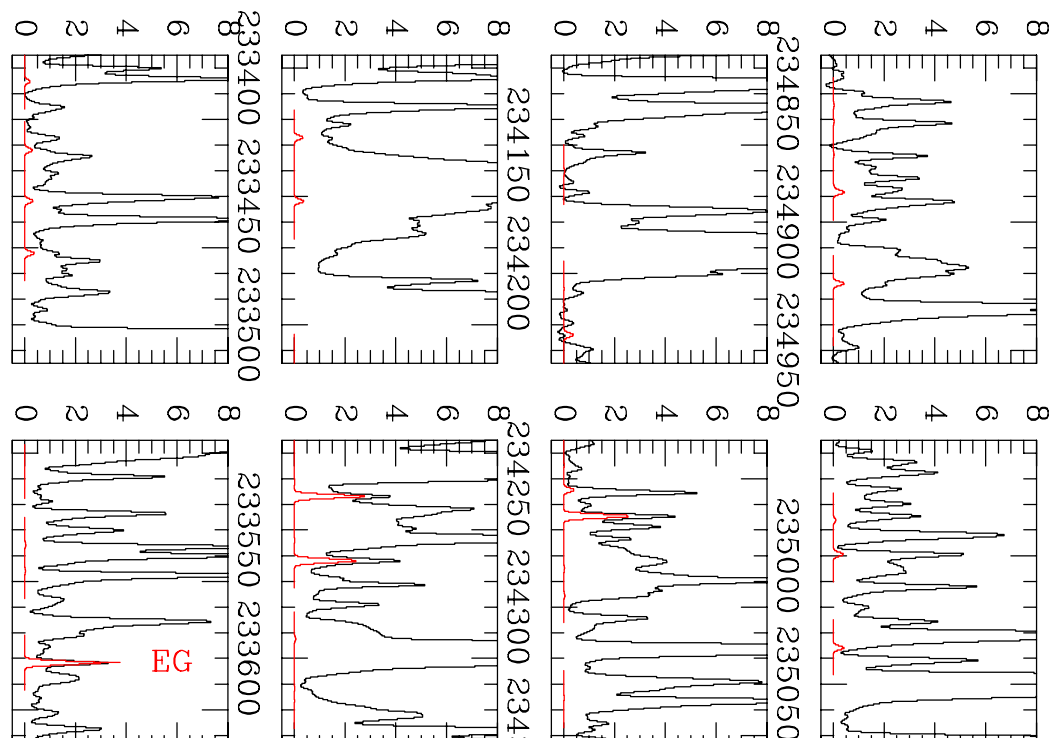

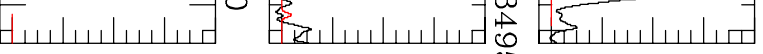
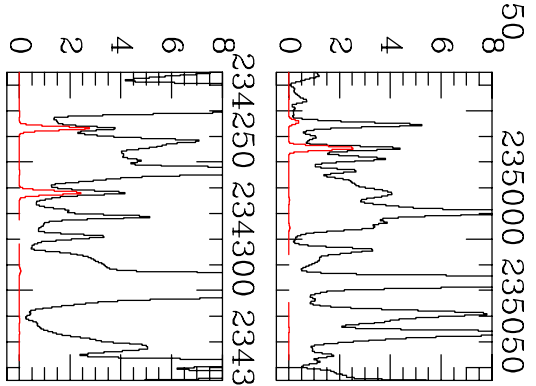

- N A O D

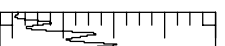

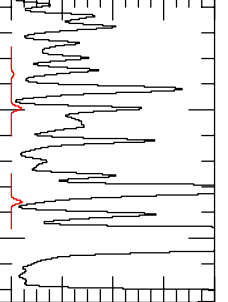

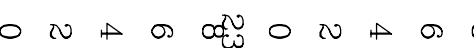
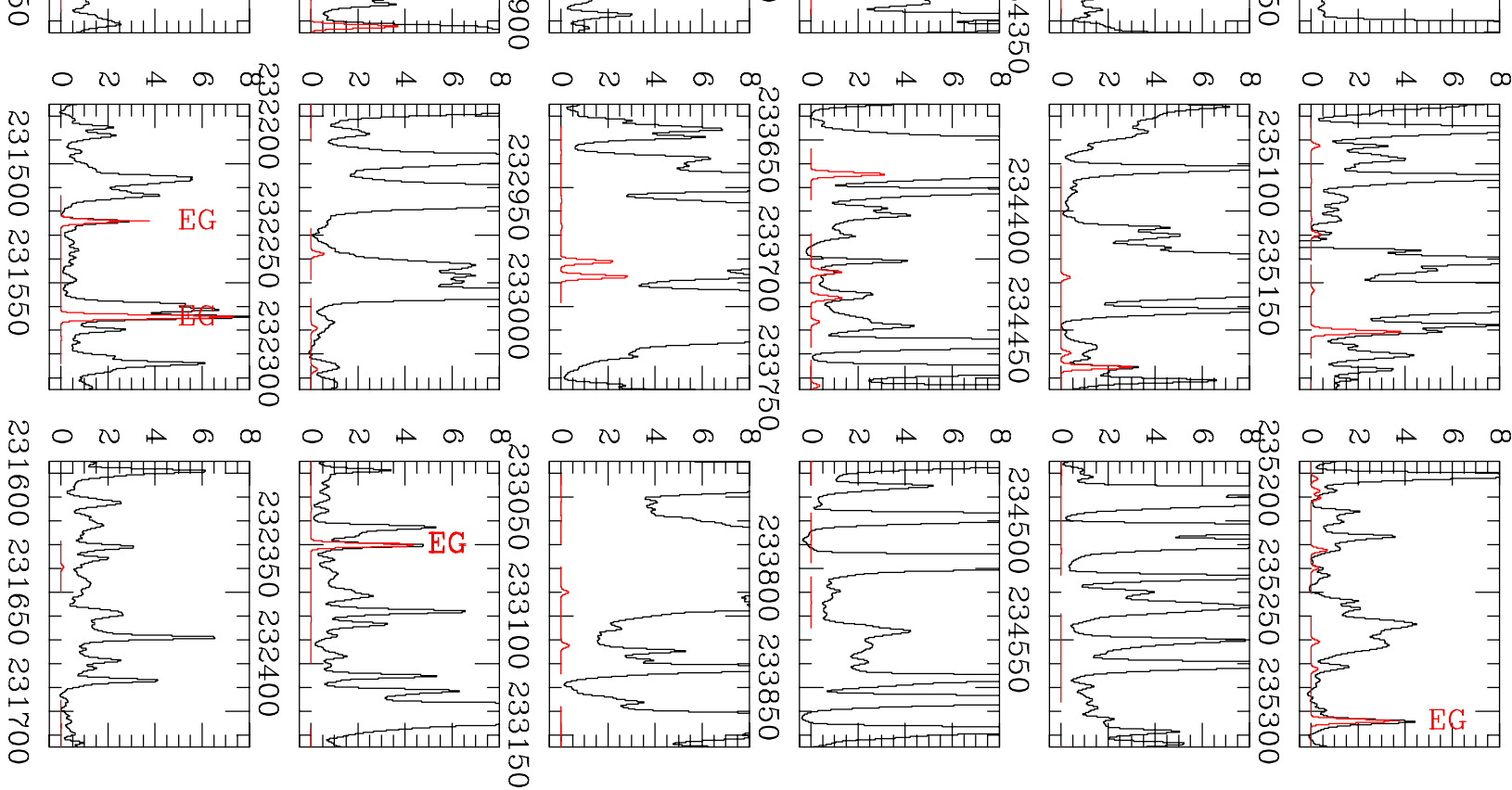

O $v a \infty \infty$
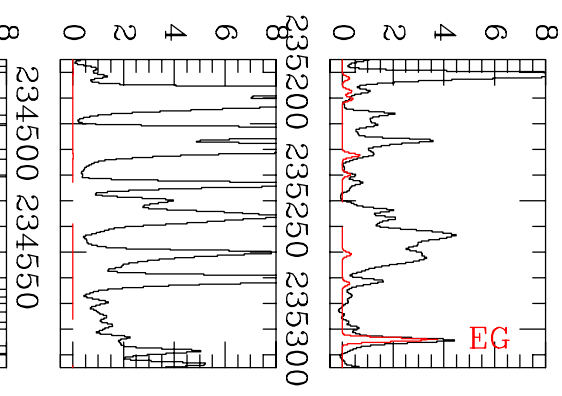

Fig. A.1. continued. 

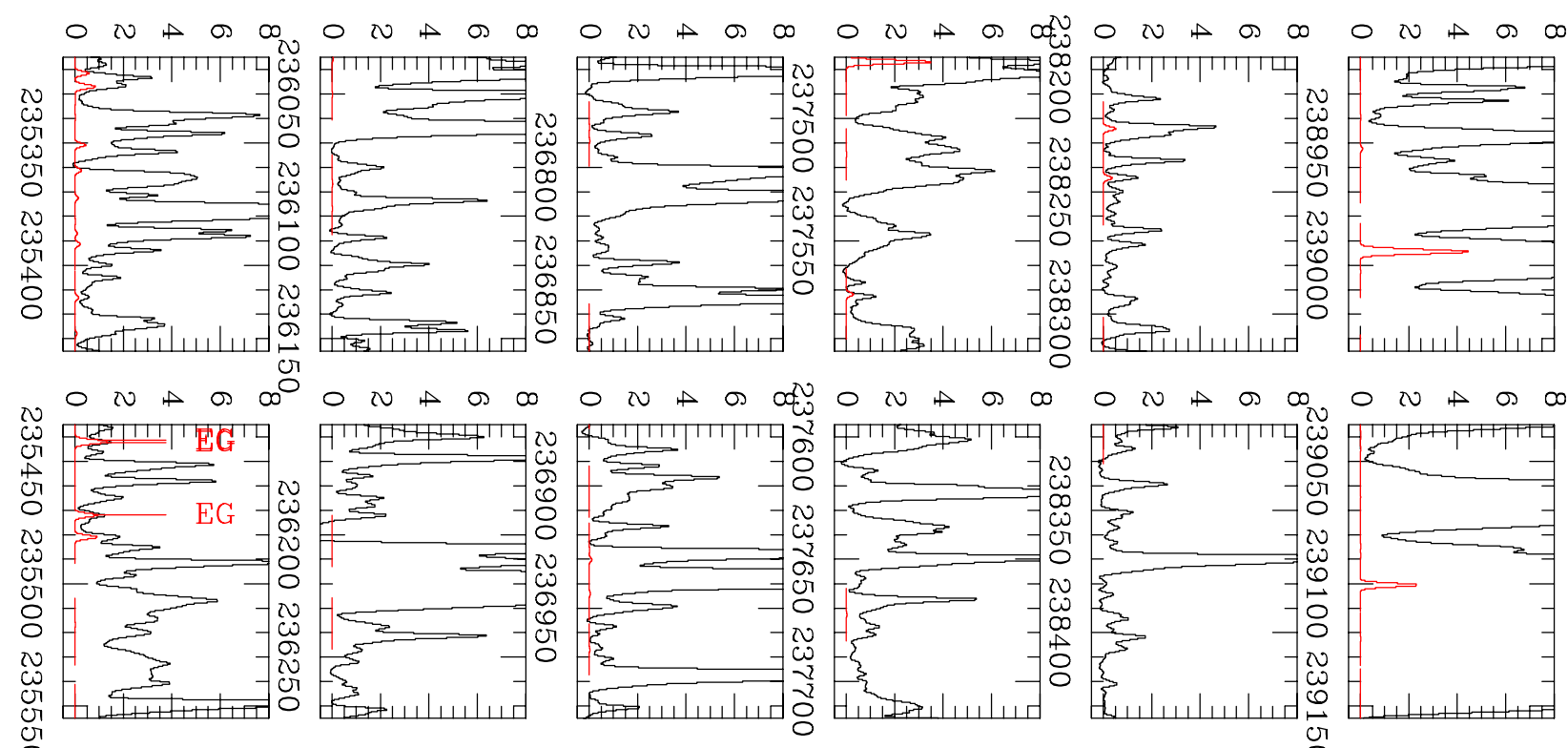

잉

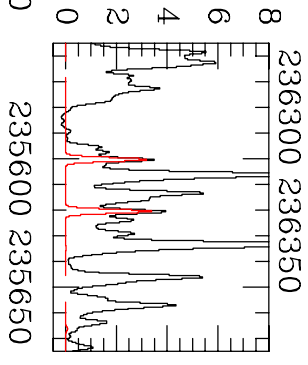

- va a a o va a

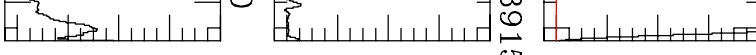

va 0 a 0 a $0 \infty^{\circ}$

o v $\begin{array}{lllllll}0 & 0 & 0 & 0\end{array}$
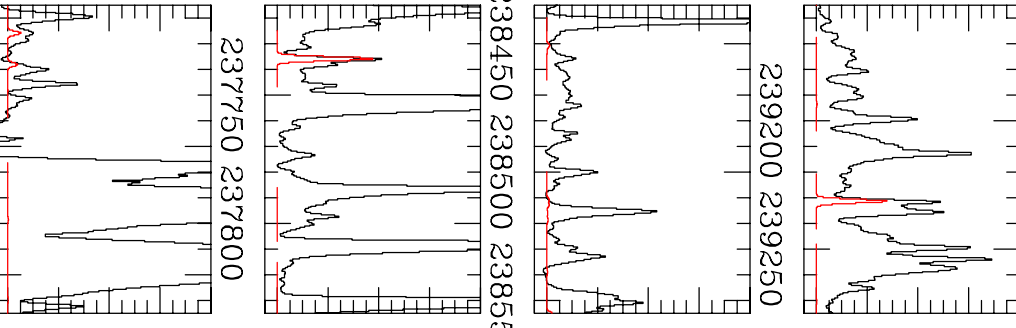

- na a d a n a a
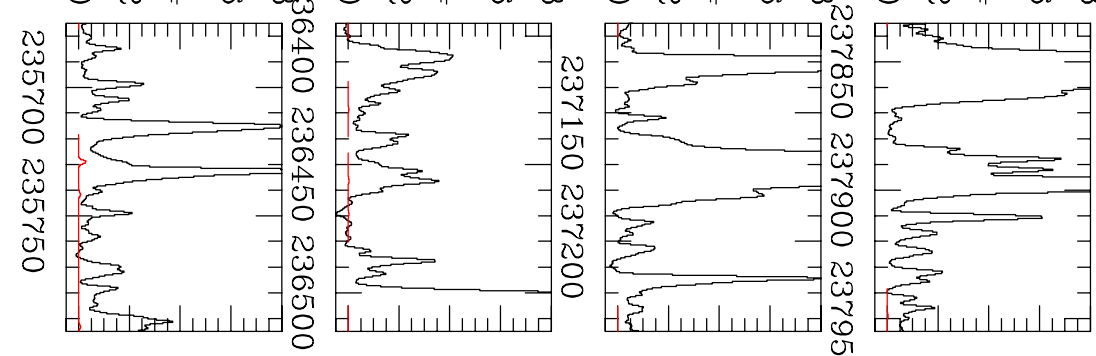

o v a a $\infty$ o v a a
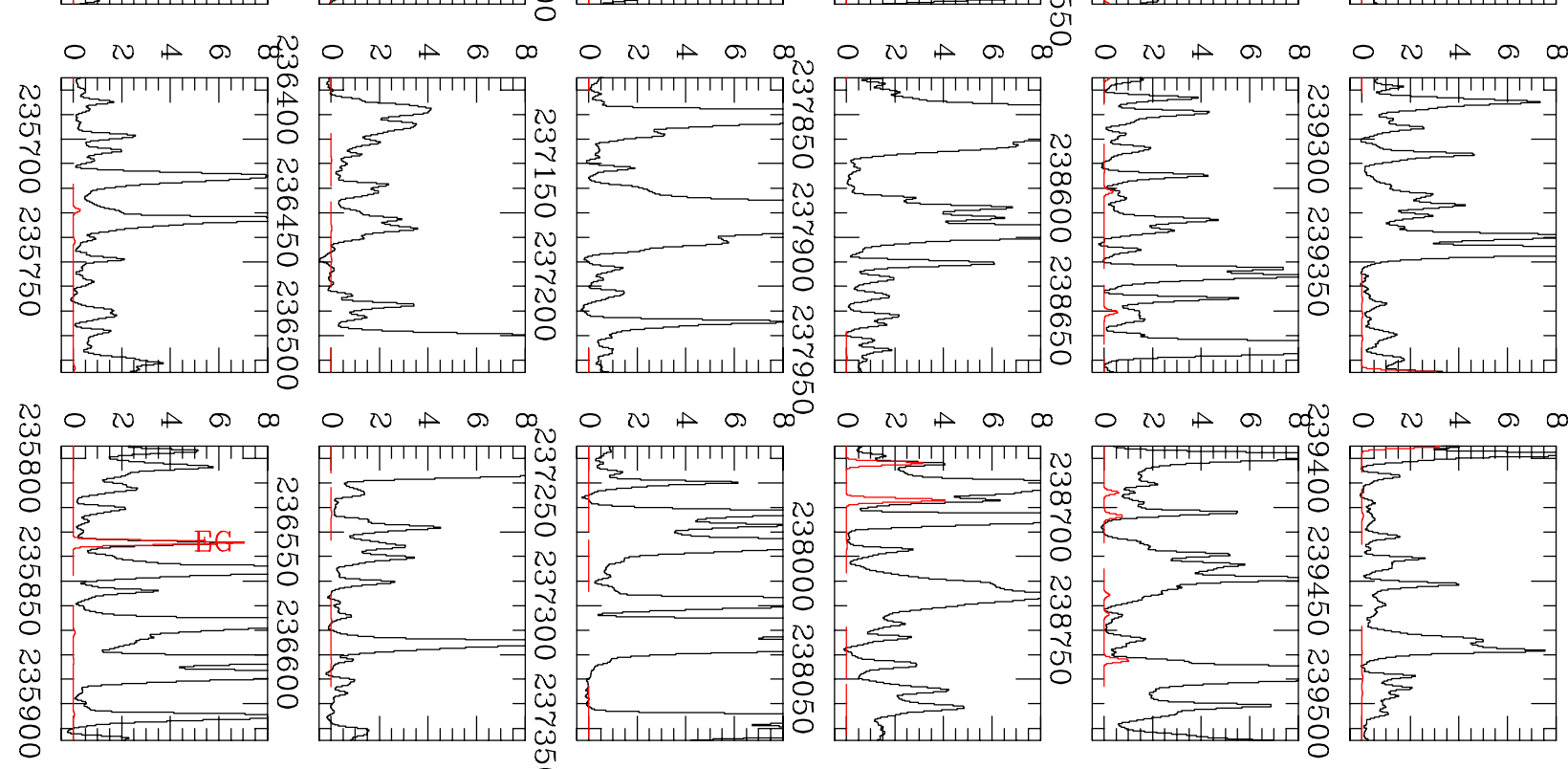

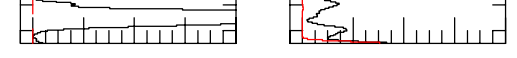
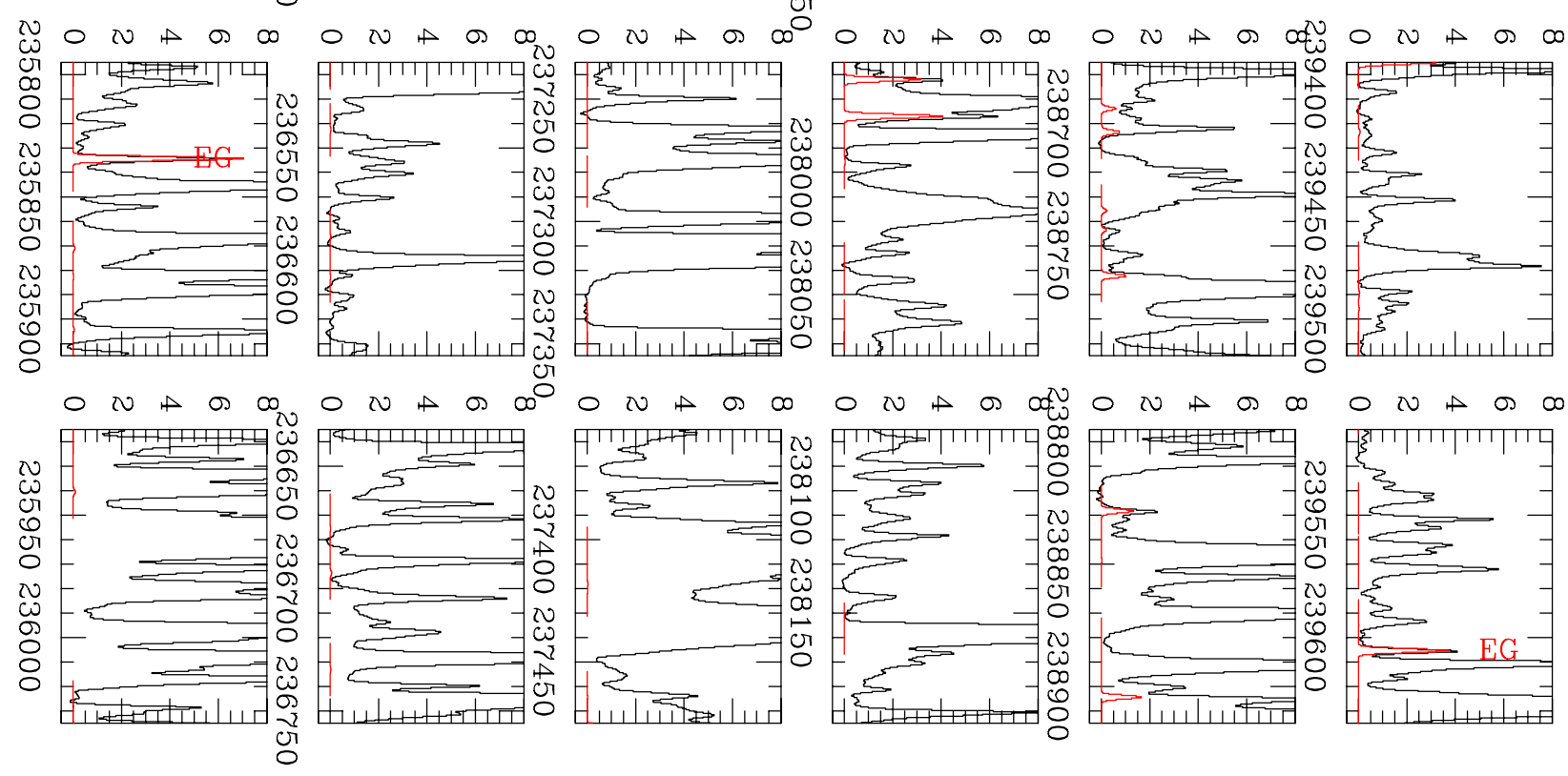

o va a $\infty$

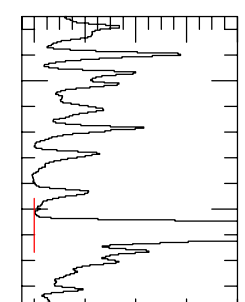

$=5$

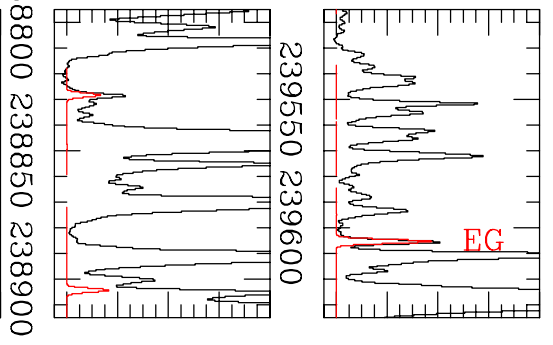

Fig. A.1. continued. 
N. Brouillet et al.: Antifreeze in the hot core of Orion
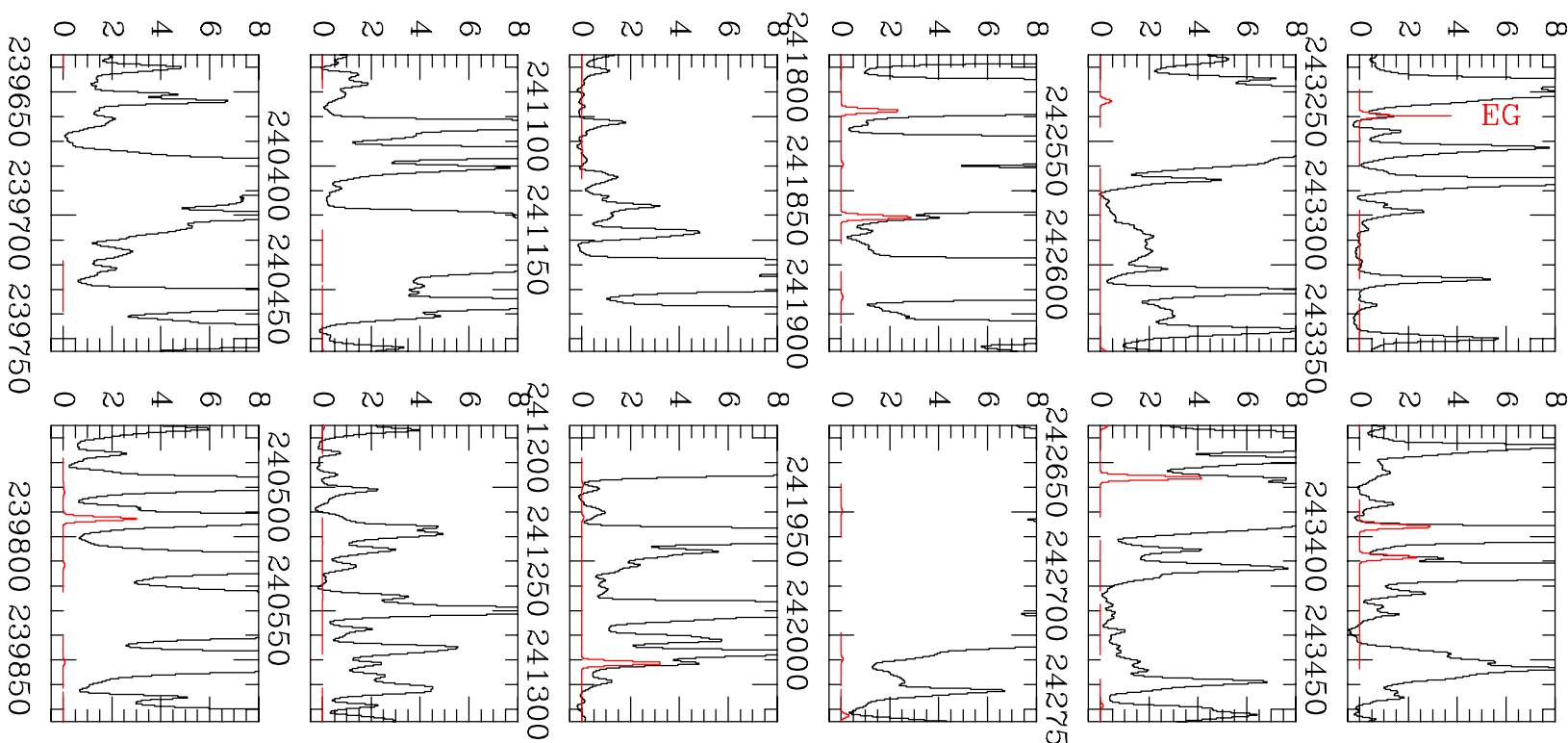

ता

- v
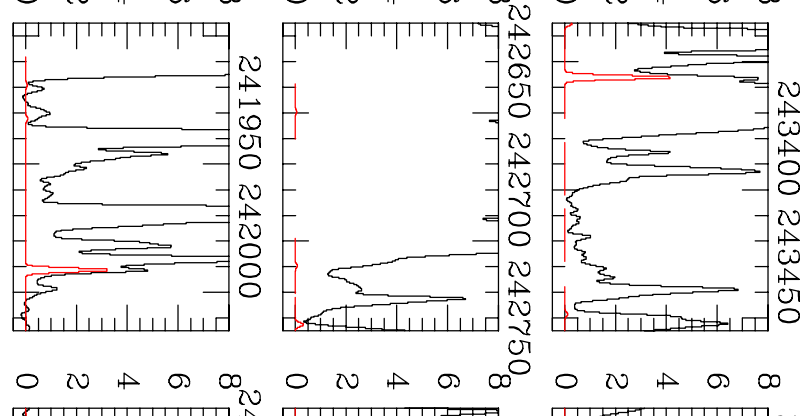

- v $A$ a $\infty$
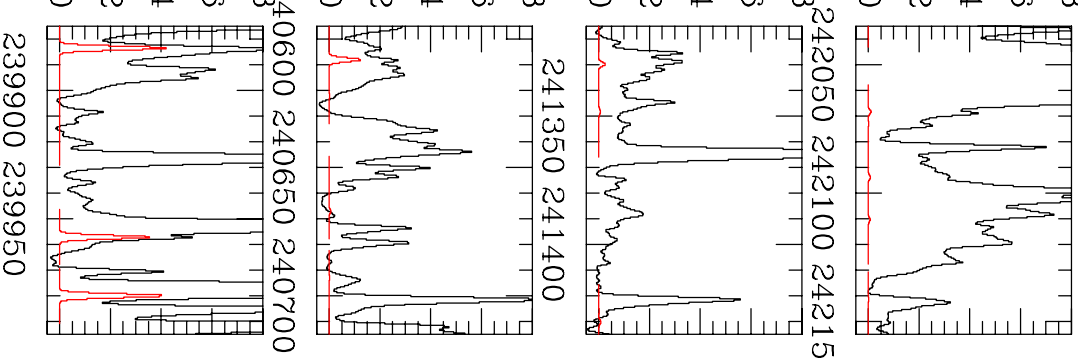

- va a $\infty$
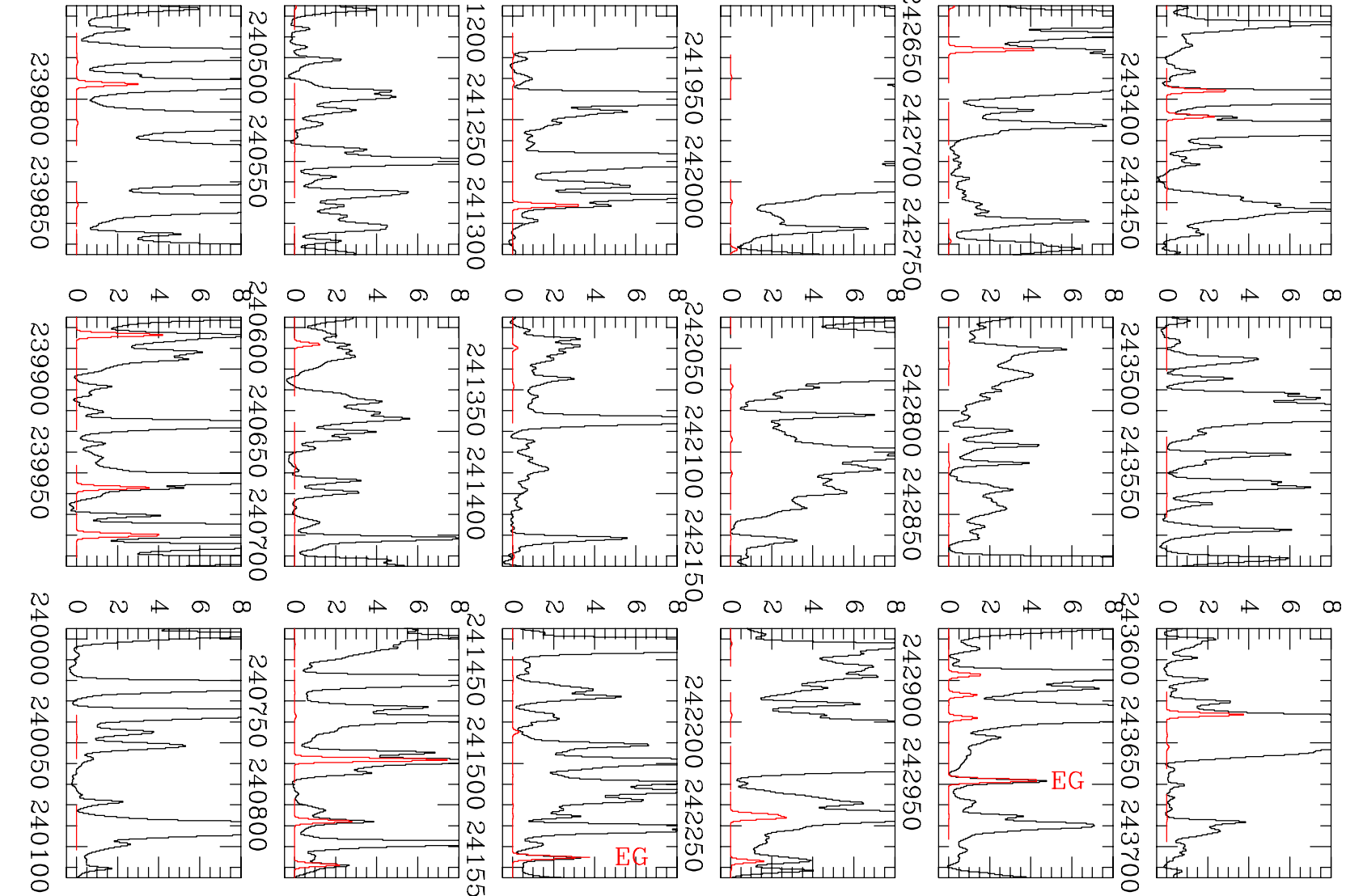

D
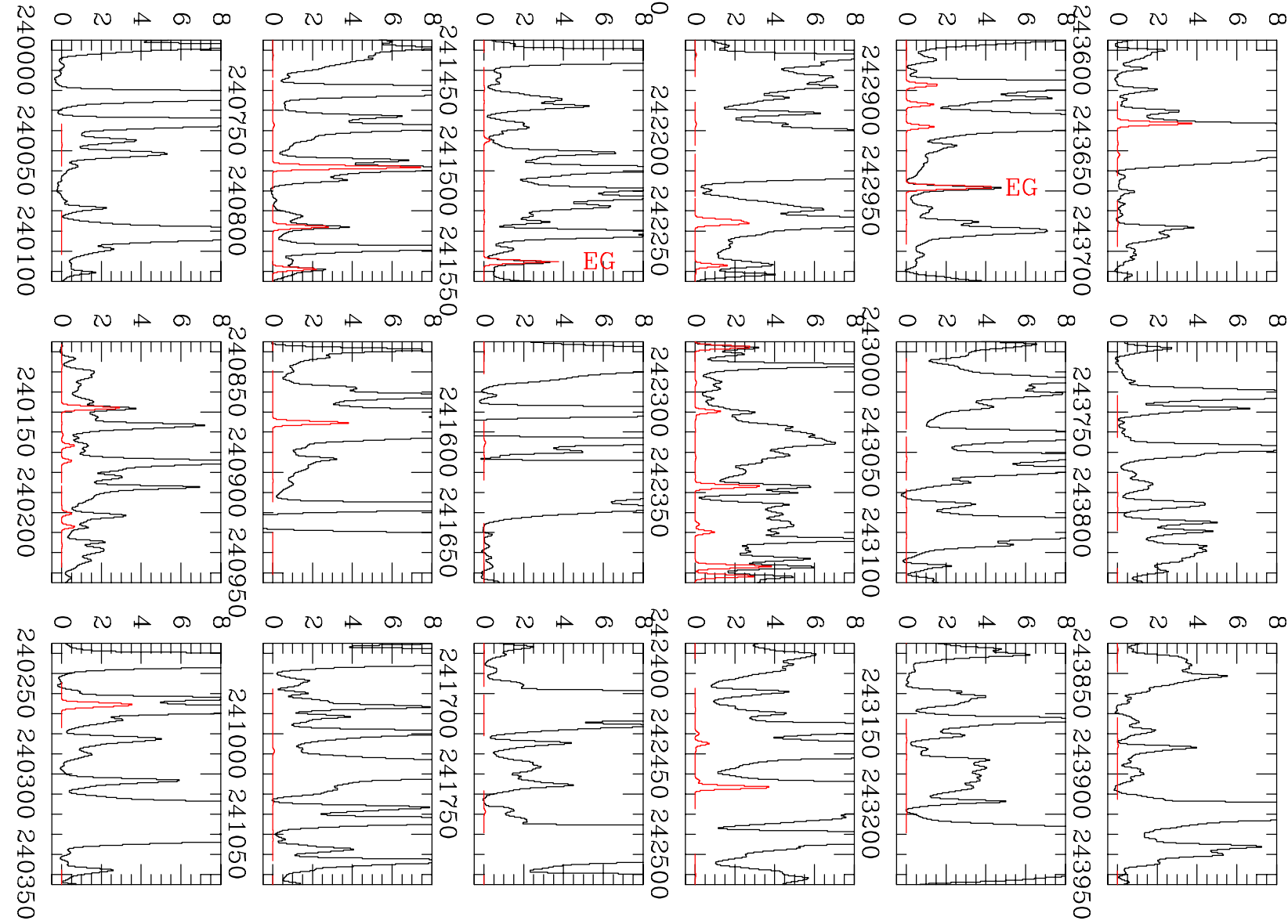

Fig. A.1. continued.

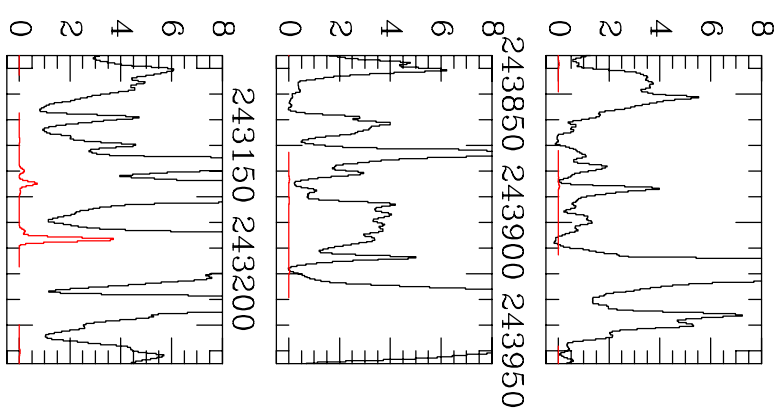


A\&A 576, A129 (2015)

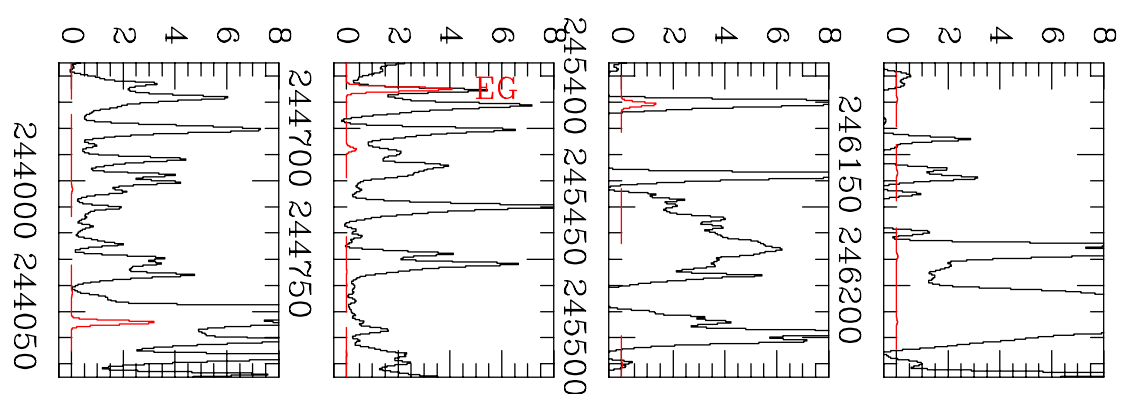

O v

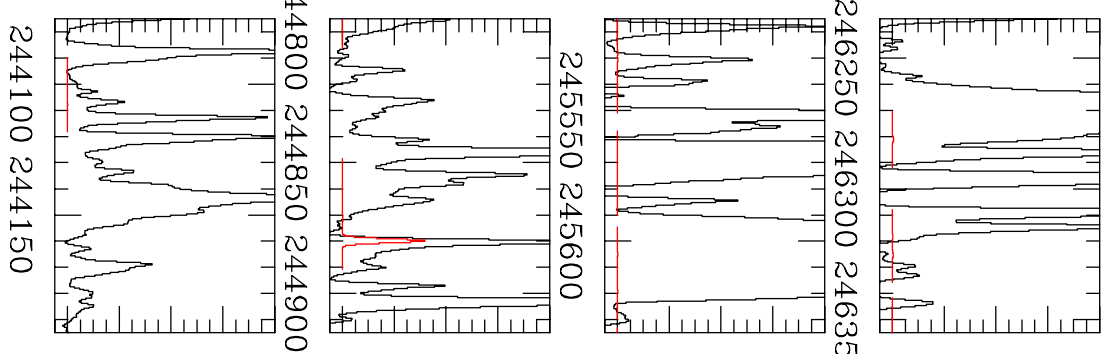

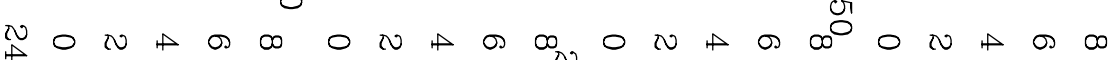

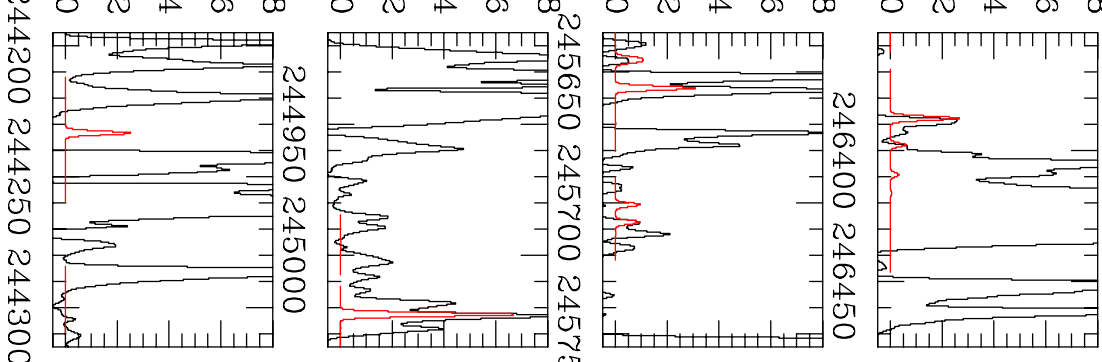

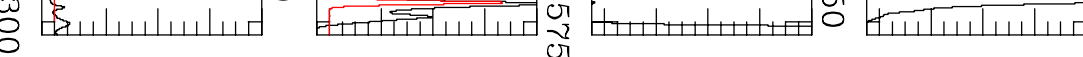

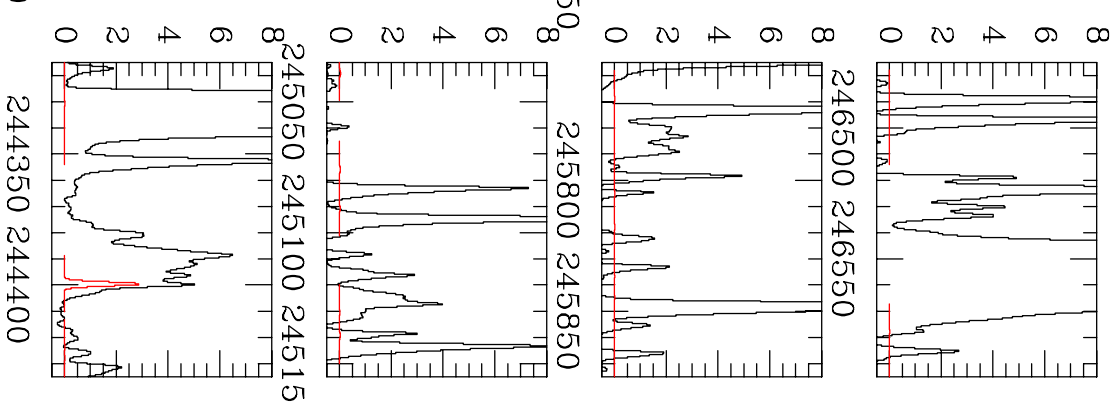

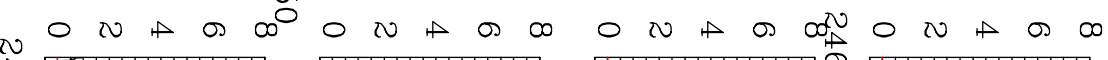

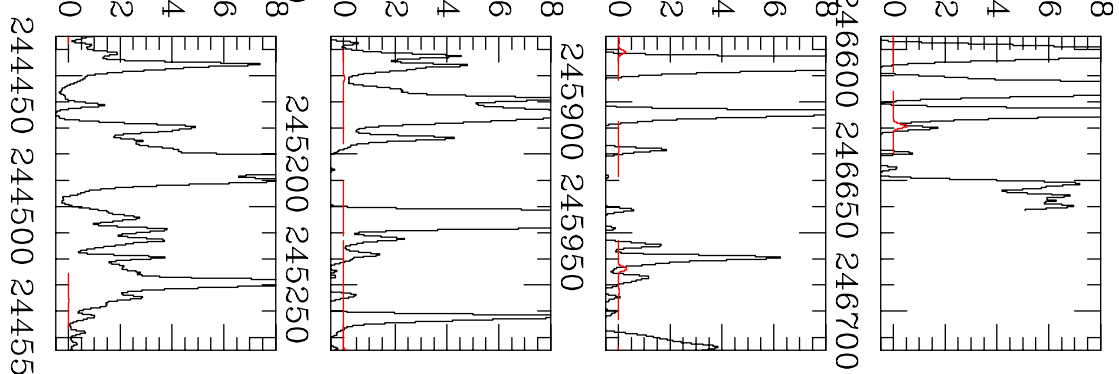

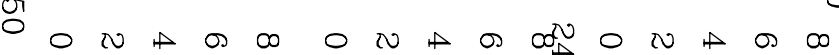

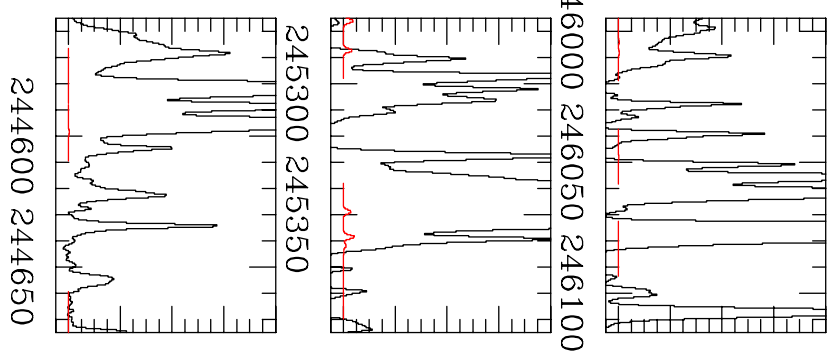

Fig. A.1. continued. 